This version April 5, 2006.

\title{
On the future infimum of positive self-similar Markov processes.
}

\author{
J.C. Pardo ${ }^{1}$ \\ ${ }^{1}$ Laboratoire de Probabilités et Modèles Aléatoires, Université Pierre et Marie Curie, 4, Place Jussieu \\ - 75252 PARIS Cedex 05. E-mail: pardomil@ccr.jussieu.fr \\ Abstract We establish integral tests and laws of the iterated logarithm for the upper envelope of \\ the future infimum of positive self-similar Markov processes and for increasing self-similar Markov \\ processes at 0 and $+\infty$. Our proofs are based on the Lamperti representation and time reversal ar- \\ guments due to Chaumont and Pardo [9]. These results extend laws of the iterated logarithm for the \\ future infimum of Bessel processes due to Khoshnevisan et al. [11].
}

Key words: Future infimum process, Self-similar Markov process, Lévy process, Lamperti representation, last passage time, integral test, law of the iterated logarithm.

A.M.S. Classification: $60 \mathrm{G} 18,60 \mathrm{G} 17,60 \mathrm{G}$ 51, 60 F 15.

\section{Introduction and main results}

An $\mathbb{R}_{+}$-valued Markov process $X=\left(X_{t}, t \geq 0\right)$ with càdlàg paths is a self-similar process if for every $k>0$ and every initial state $x \geq 0$ it satisfies the scaling property, i.e., for some $\alpha>0$

$$
\text { the law of }\left(k X_{k^{-\alpha} t}, t \geq 0\right) \text { under } \mathbb{P}_{x} \text { is } \mathbb{P}_{k x},
$$

where $\mathbb{P}_{x}$ denotes the law of the process $X$ starting from $x \geq 0$.

We will refer to positive self-similar Markov processes as PSSMP. We will also denote by $X^{(x)}$ for the PSSMP starting from $x \geq 0$. Well-known examples of this kind of processes are: Bessel processes, stable subordinators and stable Lévy processes conditioned to stay positive.

In this paper, we are interested in the class of processes which drift towards $+\infty$. Let

\footnotetext{
${ }^{1}$ Research suported by a grant from CONACYT (Mexico).
} 
$X^{(x)}$ be a PSSMP starting at $x \geq 0$ which drifts towards $+\infty$. We introduce the so-called future infimum of $X^{(x)}$, by

$$
J_{t}^{(x) \stackrel{(\text { def })}{=}} \inf _{s \geq t} X_{s}^{(x)}, \quad \text { for } \quad t \geq 0
$$

Note that the future infimum process $J^{(x)}=\left(J_{t}^{(x)}, t \geq 0\right)$, is an increasing self-similar process with the same scaling coefficient as $X^{(x)}$. It is clear that when the PSSMP $X^{(x)}$ starts from $x=0$, the process $J^{(0)}$ starts also from 0. When the PSSMP $X^{(x)}$ starts from $x>0$, the future infimum $J^{(x)}$ starts from the global infimum, that is from $\inf _{t \geq 0} X_{t}^{(x)}$. In both cases, the future infimum process $J^{(x)}$ tends to $+\infty$ as $t$ increases. We are interested in describing the upper envelope at 0 and at $+\infty$ of the future infimum process for a large class of PSSMP through integral tests and laws of iterated logarithm. As we will note later, the same integral tests will also allow us to describe the upper envelope at 0 and $+\infty$ of the PSSMP $X^{(0)}$ in the increasing case.

Khoshnevisan et al. [1] studied the asymptotic behaviour of the future infimum of some stochastic processes, in particular the case of a Bessel process with index $d>2$. They obtained the following integral test:

Let $\phi(t)=\sqrt{t} \psi(t)$ be nondecreasing in $t>0$ and assume that $\psi(t)$ diverges to $+\infty$ as $t$ goes to $+\infty$. If

$$
\int^{+\infty}(\psi(t))^{d-2} \exp \left\{-\psi^{2}(t) / 2\right\} \mathrm{d} t<+\infty
$$

then, for all $\epsilon>0$

$$
\mathbb{P}_{0}\left(J_{t}>(1+\epsilon) \phi(t) \text {, i.o., as } t \rightarrow+\infty\right)=0 .
$$

They also obtained the following law of the iterated logarithm,

$$
\limsup _{t \rightarrow+\infty} \frac{J_{t}}{\sqrt{2 t \log \log (t)}}=1, \quad \mathbb{P}_{0}-\text { a.s. }
$$

In this paper, we will give integral tests for $J$ in the general case.

Our arguments are based in the following representation of self-similar Markov processes well-known as Lamperti representation. Lamperti studied in detail the PSSMP in [12. In his main result, Lamperti proved that any PSSMP starting from a strictly positive state is a time-change of the exponential of a Lévy process. More precisely, let $X^{(x)}$ be a self-similar Markov process started from $x>0$ that fulfills the scaling property for some $\alpha>0$, then there exists $\xi=\left(\xi_{t}, t \geq 0\right)$ a Lévy process possibly killed at an independent exponential time, such that

$$
X_{t}^{(x)}=x \exp \left\{\xi_{\tau\left(t x^{-\alpha}\right)}\right\}, \quad 0 \leq t \leq x^{\alpha} I(\xi),
$$

where

$$
\tau_{t}=\inf \left\{s \geq 0: I_{s}(\xi)>t\right\}, \quad I_{s}(\xi)=\int_{0}^{s} \exp \left\{\alpha \xi_{u}\right\} \mathrm{d} u \quad \text { and } \quad I(\xi)=\lim _{t \rightarrow+\infty} I_{t}(\xi) .
$$

Lamperti raised the question of whether one can make sense of $X^{(x)}$ started from $0+$. This problem was first solved for the increasing case by Bertoin and Caballero [2, this 
is when its associated Lévy process is a subordinator. In the same work, they also computed the entrance law at 0 which will be written below. Later, Bertoin and Yor [4] studied and computed the entrance law in a more general case, when $\xi$ satisfies the following condition

$$
\text { (H) } \quad 0<\mathbb{E}\left(\xi_{1}\right) \leq \mathbb{E}\left(\left|\xi_{1}\right|\right)<\infty \quad \text { and } \quad \xi_{1} \quad \text { is not arithmetic. }
$$

It was proved in [4] that under condition $(\mathrm{H})$, the process $X^{(x)}$ converges in the sense of finite dimensional distributions. If we denote by $\mathbb{P}_{0}$ its limit law then the entrance law can be expressed as follows: for every measurable function $f: \mathbb{R}_{+} \rightarrow \mathbb{R}_{+}$and every $t>0$,

$$
\mathbb{E}_{0}\left(f\left(X_{t}\right)\right)=\frac{1}{m} \mathbb{E}\left(I(\hat{\xi})^{-1} f\left(t I(\hat{\xi})^{-1}\right)\right)
$$

where $m=E\left(\xi_{1}\right)$ and $\hat{\xi}=-\xi$. Recently Caballero and Chaumont 7] gave necessary and sufficient conditions for the weak convergence of $X^{(x)}$ on the Skorokhod's space. In the mentioned study, they give a path construction of $X^{(0)}$.

In this paper, we suppose that the limiting process $X^{(0)}$ exists in the sense of weak convergence on the Skorokhod's space and that it satisfies that $\lim _{t \rightarrow+\infty} X_{t}^{(0)}=+\infty$ which is equivalent, according to [7] to the fact that $\xi$ satisfies condition $(\mathrm{H})$. Let $\mathcal{D}$ denote the Skorokhod's space of càdlàg paths with real values and defined on $[0,+\infty)$ and $\mathbb{P}$ a probability measure defined on $\mathcal{D}$. We denote by $\mathbb{P}_{x}$, for $x>0$ the law, under $\mathbb{P}$, of the process $X^{(x)}$ defined above in (1.1) and by $\mathbb{P}_{0}$ the law, under $\mathbb{P}$, of the limiting process $X^{(0)}$ whose entrance law is given by (1.2). With this notation we have that $\left(X, \mathbb{P}_{x}\right)=\left(X^{(x)}, \mathbb{P}\right)$ for $x \geq 0$. Throughout this work we will suppose that $\xi$ is a Lévy process satisfying condition $(\mathrm{H})$.

Note that from the scaling property, the process $\left(X^{\alpha}, \mathbb{P}_{x}\right)$, for $x \geq 0$ is a PSSMP whose scaling coefficient is equal to 1 . Henceforth, without loss of generality we can assume that $\alpha=1$.

Some general results for the lower envelope of $X^{(x)}$ have been established by Chaumont and Pardo in [9]. These results are based on the study of the last passage times of the process $X^{(x)}$. Since its future infimum process $J^{(x)}$ can be seen as the right inverse of the last passage times of $X^{(x)}$, it is not difficult to deduce that we can replace $X^{(x)}$ by its future infimum in all their results. In other words, we will obtain the same integral tests for the lower envelope of $J^{(x)}$ at 0 ( when $x=0$ ) and at $+\infty$ ( for all $x \geq 0$ ). Motivated by the above and based on the study of the last passage times for the PSSMP by Chaumont and Pardo, we will describe the upper envelope of its future infimum process.

For $x>0$, we consider $\hat{X}^{(x)}$, the dual process of the PSSMP $X$ with respect to the Lebesgue measure. From Bertoin and Yor [4], we know that $\hat{X}^{(x)}$ has a Lamperti representation and is given by

$$
\hat{X}^{(x)}=\left(x \exp \left\{\hat{\xi}_{\hat{\tau}(t / x)}\right\}, 0 \leq t \leq x I(\hat{\xi})\right),
$$

where

$$
\hat{\tau}_{t}=\inf \left\{s \geq 0: I_{s}(\hat{\xi})>t\right\}, \quad I_{s}(\hat{\xi})=\int_{0}^{s} \exp \left\{\hat{\xi}_{t}\right\} \mathrm{d} t \quad \text { and } \quad I(\hat{\xi})=\lim _{s \rightarrow+\infty} I_{s}(\hat{\xi}) .
$$


Note, that $x I(\hat{\xi})$ is the first time at which the process $\hat{X}^{(x)}$ reaches the state 0 , that is $x I(\hat{\xi})=\inf \left\{t: \hat{X}_{t}^{(x)}=0\right\}$.

For $y \geq 0$, we define $T_{y}=\inf \left\{t: \xi_{t} \geq y\right\}$, the first passage time of the process $\xi$ over the state $y$, and $U(y)=\sup \left\{t: X_{t}^{(0)} \leq y\right\}$ the last passage time of the processes $X^{(0)}$ below $y$. Since the processes $\xi$ and $X^{(0)}$ drift towards $+\infty$, both random times are almost surely finite.

The following lemma proved by Chaumont and Pardo [9], gives a path decomposition of the process $X^{(0)}$ reversed at time $U(x)$, for $x>0$, and also determines the law of $U(x)$. This path decomposition consists in splitting the path of $\left(X_{t}^{(0)}, 0 \leq t \leq U(x)\right)$ at its last passage times.

Lemma 1 Let $\Gamma=X_{U(x)^{-}}^{(0)}$. Then, the process time-reversed at its last passage time below $x>0, \hat{X} \stackrel{(\text { def })}{=}\left(X_{(U(x)-t)^{-}}^{(0)}, 0 \leq t \leq U(x)\right)$ can be described as

$$
\hat{X}=\left(\Gamma \exp \left\{\hat{\xi}_{\hat{\tau}(t / \Gamma)}\right\}, 0 \leq t \leq U(x)\right)
$$

where $\Gamma$ and $\hat{\xi}$ are independent. Moreover, let $\left(x_{n}\right)$ be a decreasing sequence which tends to 0 and such that $x_{1}=x$. If we define $\hat{S}_{y}=\inf \left\{t: \hat{X}_{t} \leq y\right\}$, for $y>0$, then we can describe the process $\hat{X}$ between the passage times $\hat{S}_{x_{n}}$ and $\hat{S}_{x_{n+1}}$ as follows:

$$
\left(\hat{X}_{\hat{S}_{x_{n}}+t}, 0 \leq t \leq \hat{S}_{x_{n+1}}-\hat{S}_{x_{n}}\right)=\left(\Gamma_{n} \exp \left\{\hat{\xi}_{\hat{\tau}^{(n)}\left(t / \Gamma_{n}\right)}^{(n)}\right\}, 0 \leq t \leq H_{n}\right), \quad n \geq 1
$$

where the processes $\hat{\xi}^{(n)}, n \geq 1$ are independent between themselves and have the same law as $\hat{\xi}$ and

$$
\begin{aligned}
\hat{\tau}_{t}^{(n)} & =\inf \left\{s: I_{s}\left(\hat{\xi}^{(n)}\right)>t\right\}, \\
H_{n} & =\Gamma_{n} \int_{0}^{\hat{T}^{(n)}\left(\log \left(x_{n+1} / \Gamma_{n}\right)\right)} \exp \left\{\hat{\xi}_{s}^{(n)}\right\} \mathrm{d} s, \\
\Gamma_{n+1} & =\Gamma_{n} \exp \left\{\hat{\xi}_{\hat{T}^{(n)}\left(\log \left(x_{n+1} / \Gamma_{n}\right)\right)}\right\}, \quad n \geq 1, \quad \Gamma_{1}=\Gamma, \\
\hat{T}_{z}^{(n)} & =\inf \left\{t: \hat{\xi}_{t}^{(n)} \leq z\right\} .
\end{aligned}
$$

For each $n \geq 1, \Gamma_{n}$ is independent of $\xi^{(n)}$ and

$$
x_{n}^{-1} \Gamma_{n} \stackrel{(d)}{=} x^{-1} \Gamma
$$

where the latter equality means that both variables have the same distribution.

As a consequence, we have that for all $n \geq 1$,

$$
U\left(x_{n}\right)=\sum_{k \geq n} \Gamma_{k} \int_{0}^{\hat{T}^{(k)}\left(\log \left(x_{k+1} / \Gamma_{k}\right)\right)} \exp \left\{\hat{\xi}_{s}^{(k)}\right\} \mathrm{d} s, \quad \text { a.s. }
$$


On the other hand, since

$$
\begin{aligned}
\left(X_{\left(U\left(x_{n}\right)-t\right)^{-}}^{(0)}, 0 \leq t \leq U\left(x_{n}\right)\right) & =\left(\hat{X}_{\hat{S}_{x_{n}+t}}, 0 \leq t \leq U\left(x_{1}\right)-\hat{S}\left(x_{n}\right)\right) \\
& =\left(\Gamma_{n} \exp \left\{\bar{\xi}_{\bar{\tau}^{(n)}\left(t / \Gamma_{n}\right)}^{(n)}\right\}, 0 \leq t \leq \Gamma_{n} I\left(\bar{\xi}^{(n)}\right)\right)
\end{aligned}
$$

where $\bar{\xi}^{(n)}$ has the same law as $\hat{\xi}$ and $\bar{\tau}^{(n)}$ is the inverse of the exponential functional $I_{s}\left(\bar{\xi}^{(n)}\right)$. Then we also have that almost surely

$$
U\left(x_{n}\right)=\Gamma_{n} I\left(\bar{\xi}^{(n)}\right) \leq x_{n} I\left(\bar{\xi}^{(n)}\right), \quad \text { where } \quad I\left(\bar{\xi}^{(n)}\right)=\int_{0}^{\infty} \exp \left\{\bar{\xi}_{t}^{(n)}\right\} \mathrm{d} t,
$$

and, $\Gamma_{n}$ and $\bar{\xi}^{(n)}$ are independent.

Note that the process $\left(\hat{\xi}_{t}^{(n)}, 0 \leq t \leq \hat{T}^{(n)}\left(\log \left(x_{n+1} / \Gamma_{n}\right)\right)\right.$ is the same as the process $\bar{\xi}^{(n)}$ killed at $\bar{T}^{(n)}\left(\log \left(x_{n+1} / \Gamma_{n}\right)\right)$, where $\bar{T}^{(n)}(x)=\inf \left\{t: \bar{\xi}_{t}^{(n)} \leq x\right\}$ for $x \leq 0$. In fact, the process $\bar{\xi}^{(n)}$ can be described as follows,

$$
\bar{\xi}_{t}^{(n)}= \begin{cases}\hat{\xi}_{t}^{(n)} & \text { if } t \in\left[0, \Sigma_{1}^{(n)}[,\right. \\ \hat{\xi}_{t-\Sigma_{1}^{(n)}}^{(n+1)} & \text { if } t \in\left[\Sigma_{1}^{(n)}, \Sigma_{2}^{(n)}[,\right. \\ \vdots & \\ \hat{\xi}_{t-\Sigma_{k}^{(n)}}^{(n+k)} & \text { if } t \in\left[\Sigma_{k}^{(n)}, \Sigma_{k+1}^{(n)}[,\right. \\ \vdots & \end{cases}
$$

where $\Sigma_{k}^{(n)}=\sum_{j=n}^{n+k-1} \hat{T}^{(j)}$ and $\hat{T}^{(j)}=\hat{T}^{(j)}\left(\log \left(x_{j+1} / \Gamma_{j}\right)\right)$.

Chaumont and Pardo proved in the same work that we have the same properties for $x$ large (see Corollary 3 in [9]). This will be very useful to establish our asymptotic results at $+\infty$.

It is important to note that the law of $x_{1}^{-1} \Gamma$ is related with the upward ladder height process $\sigma=\left(\sigma_{t}, t \geq 0\right)$ associated to $\xi$ (see Bertoin 1 for a proper definition). In fact, its law (see for instance Lemma 1 in 9] ) is the same as that of $\exp \{-U Z\}$, where $U$ and $Z$ are independent random variables, $U$ is uniformly distributed over $[0,1]$ and the law of $Z$ is given by

$$
\mathbb{P}(Z>u)=\mathbb{E}\left(\sigma_{1}\right)^{-1} \int_{(u, \infty)} s \mu(\mathrm{d} s), \quad u \geq 0
$$

where $\mu$ is the Lévy measure of $\sigma$. In particular, we can deduce that for all $y<x_{1}$, $\mathbb{P}(\Gamma>y)>0$.

The following result gives us integral tests at 0 for the upper envelope of $J^{(0)}$. This theorem means in particular that the asymptotic behaviour of $J^{(0)}$ only depends on the tail behaviour of the law of $\nu I(\hat{\xi})$ and this of $I(\hat{\xi})$, where $\nu$ is independent of $I(\hat{\xi})$ and has the same distribution as $x_{1}^{-1} \Gamma$. Note that the support of the law of $\nu$ is the interval $[0,1]$.

Let us define

$$
\bar{F}_{\nu}(t) \stackrel{(\text { def) }}{=} \mathbb{P}(\nu I(\hat{\xi})<t) \text { and } \bar{F}(t) \stackrel{\text { (def) }}{=} \mathbb{P}(I(\hat{\xi})<t)
$$

and denote by $\mathcal{H}_{0}$ the totality of positive increasing functions $h(t)$ on $(0, \infty)$ that satisfy 
i) $h(0)=0$, and

ii) there exists $\beta \in(0,1)$ such that $\sup _{t<\beta} \frac{t}{h(t)}<\infty$.

Theorem 1 Let $h \in \mathcal{H}_{0}$.

i) If

$$
\int_{0^{+}} \bar{F}_{\nu}\left(\frac{t}{h(t)}\right) \frac{\mathrm{d} t}{t}<\infty
$$

then for all $\epsilon>0$

$$
\mathbb{P}_{0}\left(J_{t}>(1+\epsilon) h(t), \text { i.o., as } t \rightarrow 0\right)=0 .
$$

ii) If

$$
\int_{0^{+}} \bar{F}\left(\frac{t}{h(t)}\right) \frac{\mathrm{d} t}{t}=\infty,
$$

then for all $\epsilon>0$

$$
\mathbb{P}_{0}\left(J_{t}>(1-\epsilon) h(t), \text { i.o., as } t \rightarrow 0\right)=1 .
$$

For the integral tests at $+\infty$, we define $\mathcal{H}_{\infty}$, the totality of positive increasing functions $h(t)$ on $(0, \infty)$ that satisfy

i) $\lim _{t \rightarrow \infty} h(t)=\infty$, and

ii) there exists $\beta>1$ such that $\sup _{t>\beta} \frac{t}{h(t)}<\infty$.

Then the upper envelope of $J^{(x)}$ at $+\infty$ is given by the following result.

Theorem 2 Let $h \in \mathcal{H}_{\infty}$.

i) If

$$
\int^{+\infty} \bar{F}_{\nu}\left(\frac{t}{h(t)}\right) \frac{\mathrm{d} t}{t}<\infty,
$$

then for all $\epsilon>0$ and for all $x \geq 0$,

$$
\mathbb{P}_{x}\left(J_{t}>(1+\epsilon) h(t), \text { i.o., as } t \rightarrow+\infty\right)=0 .
$$

ii) If

$$
\int^{+\infty} \bar{F}\left(\frac{t}{h(t)}\right) \frac{\mathrm{d} t}{t}=\infty,
$$

then for all $\epsilon>0$ and for all $x \geq 0$

$$
\mathbb{P}_{x}\left(J_{t}>(1-\epsilon) h(t), \text { i.o., as } t \rightarrow+\infty\right)=1 \text {. }
$$


The rest of this paper is organized as follows. In section 2, we state two Theorems that describe the lower envelope of the last passage time process $U$ at 0 and at $+\infty$, respectively. In section 3 we prove Theorems 1 and 2. Sections 4 and 5 are devoted to the "regular" case and "logregular" case respectively and some examples. In section 6, we study the case of transient Bessel processes and finally in section 7 we discuss the upper envelope of the increasing self-similar Markov processes.

\section{The lower envelope of the last passage times}

Let us recall the definition of the last passage time of $X^{(0)}$,

$$
U(x)=\sup \left\{t \geq 0: X_{t}^{(0)} \leq x\right\} \quad \text { for } x \geq 0 .
$$

From this definition, we see that $U=(U(x), x \geq 0)$ is also an increasing self-similar process whose scaling coefficient is the inverse of the scaling coefficient of $X^{(0)}$. Since the process $X^{(0)}$ starts at 0 and drifts towards $+\infty$, we deduce that the process $U$ also starts at 0 and tends to infinity as $x$ increases.

In this section, we are interested in the study of the behaviour of process $U$ at 0 and at $+\infty$. As we will see in the following section, the asymptotic behaviour of process $U$ is related to the asymptotic behaviour of the future infimum of $X^{(0)}$. In fact, we will see that the lower envelope of the last passage time process $U$ determines the upper envelope of $J^{(0)}$.

The following result will give us integral tests at 0 for the lower envelope of $U$.

Let us denote by $\mathcal{H}_{0}^{-1}$ the totality of positive increasing functions $h(x)$ on $(0, \infty)$ that satisfy

i) $h(0)=0$, and

ii) there exists $\beta \in(0,1)$ such that $\sup _{x<\beta} x^{-1} h(x)<\infty$.

Theorem 3 Let $h \in \mathcal{H}_{0}^{-1}$.

i) If

$$
\int_{0^{+}} \bar{F}_{\nu}\left(\frac{h(x)}{x}\right) \frac{\mathrm{d} x}{x}<\infty,
$$

then for all $\epsilon>0$

$$
\mathbb{P}(U(x)<(1-\epsilon) h(x) \text {, i.o., as } x \rightarrow 0)=0 .
$$

ii) If

$$
\int_{0^{+}} \bar{F}\left(\frac{h(x)}{x}\right) \frac{\mathrm{d} x}{x}=\infty
$$

then for all $\epsilon>0$

$$
\mathbb{P}(U(x)<(1+\epsilon) h(x), \text { i.o., as } x \rightarrow 0)=1 .
$$


Proof: We first prove the convergent part. Let $\left(x_{n}\right)$ be a decreasing sequence of positive numbers which converges to 0 and let us define the events $A_{n}=\left\{U\left(x_{n+1}\right)<h\left(x_{n}\right)\right\}$. Now, we choose $x_{n}=r^{n}$, for $r<1$. From the first Borel Cantelli's Lemma, if $\sum_{n} \mathbb{P}\left(A_{n}\right)<\infty$, it follows

$$
U\left(r^{n+1}\right) \geq h\left(r^{n}\right) \quad \mathbb{P}-\text { a.s. }
$$

for all large $n$. Since the function $h$ and the process $U$ are increasing, we have

$$
U(x) \geq h(x) \quad \text { for } \quad r^{n+1} \leq x \leq r^{n} .
$$

From (1.10), we get the following inequality

$$
\begin{aligned}
\sum_{n} \mathbb{P}\left(U\left(r^{n}\right)<h\left(r^{n+1}\right)\right) & \leq \int_{1}^{\infty} \mathbb{P}\left(r^{t} \nu I(\hat{\xi})<h\left(r^{t}\right)\right) \mathrm{d} t \\
& =-\frac{1}{\log r} \int_{0}^{r} \bar{F}_{\nu}\left(\frac{h(x)}{x}\right) \frac{\mathrm{d} x}{x}
\end{aligned}
$$

From our hypothesis, this last integral is finite. Then from the above discussion, there exist $x_{0}$ such that for every $x \geq x_{0}$

$$
U(x) \geq r^{2} h(x), \quad \text { for all } \quad r<1 .
$$

Clearly, this implies that

$$
\mathbb{P}\left(U(x)<r^{2} h(x) \text {, i.o., as } x \rightarrow 0\right)=0,
$$

which proves part $(i)$.

Now we prove the divergent part. First, note that when $\xi$ has no positive jumps the process $U$ is like the ones considered in Watanabe's work, that is $U$ is an increasing self-similar process with independent increments, but in the general case the process $U$ does not have this property. The decomposition (1.8) and the a.s. equality in (1.10) will allow us to extend the arguments used by Watanabe to our case.

Now, we assume that $h$ satisfies

$$
\int_{0^{+}} \bar{F}\left(\frac{h(x)}{x}\right) \frac{\mathrm{d} x}{x}=\infty .
$$

Let us take, again $x_{n}=r^{n}$ for $r<1$, and define the events

$$
C_{n}=\left\{U(x)<r^{-2} h(x), \text { for some } x \in\left(0, r^{n}\right)\right\} .
$$

Note that the family $\left(C_{n}\right)$ is decreasing, then

$$
C=\bigcap_{n \geq 1} C_{n}=\left\{U(x)<r^{-2} h(x), \text { i.o., as } x \rightarrow 0\right\} .
$$


If we prove that $\lim \mathbb{P}\left(C_{n}\right)>0$, then since $X^{(0)}$ is a Feller process and by Blumenthal's 0-1 law we will have that

$$
\mathbb{P}\left(U(x)<r^{-2} h(x) \text {, i.o., as } x \rightarrow 0\right)=1,
$$

which will prove part $(i i)$.

In this direction, we define the following events. For $n \leq m-1$,

$$
D_{(n, m)}=\left\{r^{j+1} \bar{I}_{(j+1, m+1)} \geq h\left(r^{j}\right), \text { for all } n \leq j \leq m-1\right\}
$$

and for $r<k<1$ and $n \leq m-2$

$E_{(n, m-1)}=\left\{r^{j+1} \bar{I}_{(j+1, m)}+r^{j+1} R_{(j+1, m)} \bar{I}_{(m, m+1)} \geq h\left(r^{j}\right)\right.$, for all $\left.n \leq j \leq m-2\right\}$ and $E_{(n, m-1)}^{(k)}=\left\{r^{j+1} \bar{I}_{(j+1, m)}+r^{j+1} R_{(j+1, m)} \bar{I}_{m}^{(k)} \geq h\left(r^{j}\right)\right.$, for all $\left.n \leq j \leq m-2\right\}$,

where

$$
\begin{aligned}
\bar{I}_{(j+1, m+1)} & =\int_{0}^{\bar{T}^{(j+1)}\left(\log \left(r^{m+1} / \Gamma_{j+1}\right)\right)} \exp \left\{\bar{\xi}_{s}^{(j+1)}\right\} \mathrm{d} s, \\
\bar{I}_{m}^{(k)} & =\int_{0}^{\bar{T}^{(m)}\left(\log \left(r^{m+1} / k r^{m}\right)\right)} \exp \left\{\bar{\xi}_{s}^{(m)}\right\} \mathrm{d} s \quad \text { and } \\
R_{(j+1, m)} & =\exp \left\{\bar{\xi}_{\bar{T}^{(j+1)}\left(\log \left(r^{m} / \Gamma_{j+1}\right)\right)}^{(j+1)}\right\},
\end{aligned}
$$

and for $n \leq j \leq m-1, \bar{\xi}^{(j+1)}$ is a Lévy process defined as in (1.11).

From the definition of $\bar{\xi}^{(j+1)}$, we can deduce that for $j<m$

$$
\begin{aligned}
\bar{\xi}^{(m)} & =\left(\bar{\xi}_{\bar{T}^{(j+1)}\left(\log \left(r^{m} / \Gamma_{j+1}\right)\right)+t}^{(j+1)}-\bar{\xi}_{\bar{T}^{(j+1)}\left(\log \left(r^{m} / \Gamma_{j+1}\right)\right)}^{(j+1)}, t \geq 0\right) \quad \text { and } \\
\Gamma_{m} & =\Gamma_{j+1} \exp \left\{\bar{\xi}_{\bar{T}^{(j+1)}\left(\log \left(r^{m} / \Gamma_{j+1}\right)\right)}^{(j+1)}\right\},
\end{aligned}
$$

then it is straightforward that

$$
\bar{T}^{(j+1)}\left(\log \left(r^{m+1} / \Gamma_{j+1}\right)\right)=\bar{T}^{(j+1)}\left(\log \left(r^{m} / \Gamma_{j+1}\right)\right)+\inf \left\{t \geq 0 ; \bar{\xi}_{t}^{(m)} \leq \log \left(r^{m+1} / \Gamma_{m}\right)\right\} .
$$

The above decomposition allows us to determine the following identity

$$
\bar{I}_{(j+1, m+1)}=\bar{I}_{(j+1, m)}+R_{(j+1, m)} \bar{I}_{(m, m+1)} .
$$

In the same way we can also get that,

$$
I\left(\bar{\xi}^{(j+1)}\right)=\bar{I}_{(j+1, m+1)}+R_{(j+1, m+1)} I\left(\bar{\xi}^{(m+1)}\right) .
$$

By Lemma 1 and the decomposition (1.11), it follows that $I\left(\bar{\xi}^{(m+1)}\right)$ is independent of $\left(\bar{I}_{(j+1, m+1)}, R_{(j+1, m+1)}\right)$ and distributed as $I(\hat{\xi})$.

From (2.12) and since

$$
\left\{r^{m} \bar{I}_{(m, m+1)} \geq h\left(r^{m-1}\right)\right\} \subset\left\{\Gamma_{m}>r^{m+1}\right\}
$$


we conclude that

$$
D_{(n, m)}=E_{(n, m-1)} \bigcap\left\{r^{m} \bar{I}_{(m, m+1)} \geq h\left(r^{m-1}\right)\right\} \bigcap\left\{\Gamma_{m}>r^{m+1}\right\} .
$$

Now, for $n \leq m-1$, we define

$$
H(n, m)=\mathbb{P}\left(E_{(n, m-1)}^{(k)}, r^{m} \bar{I}_{m}^{(k)} \geq h\left(r^{m-1}\right), \Gamma_{m}>r^{m} k\right) .
$$

On the event $\left\{\Gamma_{m}>r^{m} k\right\}$, we have that $\bar{I}_{m}^{(k)} \leq \bar{I}_{(m, m+1)}$. Hence since $k>r$, we deduce that $\mathbb{P}\left(D_{(n, m)}\right) \geq H(n, m)$.

For our purpose, we will prove that there exist $\left(n_{l}\right)$ and $\left(m_{l}\right)$, two increasing sequences such that $0 \leq n_{l} \leq m_{l}-1$, and $n_{l}, m_{l}$ go to $\infty$ and $H\left(n_{l}, m_{l}\right)$ tends to 0 as $l$ goes to infinity. In this direction, we define the events

$$
B_{n}=\left\{r^{n+1} I\left(\bar{\xi}^{(n+1)}\right)<h\left(r^{n}\right)\right\} .
$$

If we suppose the contrary, this is that there exists $\delta>0$ such that $H(n, m) \geq \delta$ for all sufficiently large integers $m$ and $n$, we see from identity (2.13) that

$$
\begin{aligned}
1 & \geq \mathbb{P}\left(\bigcup_{m=n+1}^{\infty} B_{m}\right) \geq \sum_{m=n+1}^{\infty} \mathbb{P}\left(B_{m} \bigcap\left(\bigcap_{j=n}^{m-1} B_{j}^{c}\right)\right) \\
& =\sum_{m=n+1}^{\infty} \mathbb{P}\left(r^{m+1} I\left(\bar{\xi}^{(m+1)}\right)<h\left(r^{m}\right), \bigcap_{j=n}^{m-1}\left\{r^{j+1} I\left(\bar{\xi}^{(j+1)}\right) \geq h\left(r^{j}\right)\right\}\right) \\
& \geq \sum_{m=n+1}^{\infty} \mathbb{P}\left(r^{m+1} I\left(\bar{\xi}^{(m+1)}\right)<h\left(r^{m}\right)\right) \mathbb{P}\left(D_{(n, m)}\right) \\
& \geq \sum_{m=n+1}^{\infty} \mathbb{P}\left(r^{m+1} I\left(\bar{\xi}^{(m+1)}\right)<h\left(r^{m}\right)\right) H(n, m) \geq \delta \sum_{m=n+1}^{\infty} \mathbb{P}\left(r^{m+1} I(\hat{\xi})<h\left(r^{m}\right)\right),
\end{aligned}
$$

but this last sum diverges, since

$$
\begin{aligned}
\sum_{m=n+1}^{\infty} \mathbb{P}\left(r^{m+1} I(\hat{\xi})<h\left(r^{m}\right)\right) & \geq \int_{n+1}^{\infty} \mathbb{P}\left(r^{t} I(\hat{\xi})<h\left(r^{t}\right)\right) \mathrm{d} t \\
& =-\frac{1}{\log r} \int_{0}^{r^{n+1}} \bar{F}\left(\frac{h(x)}{x}\right) \frac{\mathrm{d} x}{x}
\end{aligned}
$$

Hence our assertion is true.

Next, we denote $\mathbb{P}(I(\hat{\xi}) \in \mathrm{d} x)=\mu(\mathrm{d} x)$ and $\mathbb{P}\left(I_{r / k} \in \mathrm{d} x\right)=\bar{\mu}(\mathrm{d} x)$ for $k>r$, where

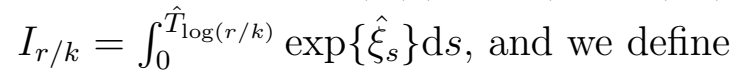

$$
\rho_{n_{l}, m_{l}}(x)=\mathbb{P}\left(\bigcap_{j=n_{l}}^{m_{l}-2}\left\{r^{j+1} \bar{I}_{\left(j+1, m_{l}\right)}+r^{j+1} R_{j+1, m_{l}} x \geq r^{-1} h\left(r^{j}\right)\right\}, \Gamma_{m_{l}}>k r^{m_{l}}\right)
$$


and

$$
G\left(n_{l}, m_{l}\right)=\mathbb{P}\left(\bigcap_{j=n_{l}}^{m_{l}-1}\left\{r^{j+1} I\left(\bar{\xi}^{(j+1)}\right) \geq h\left(r^{j}\right)\right\}, \Gamma_{m_{l}}>k r^{m_{l}}\right) .
$$

Note that $\rho_{n_{l}, m_{l}}(x)$ is increasing in $x$.

Hence, $H\left(n_{l}, m_{l}\right)$ and $G\left(n_{l}, m_{l}\right)$ are expressed as follows

$$
\begin{aligned}
& H\left(n_{l}, m_{l}\right)=\int_{r^{-m_{l} h\left(r^{m_{l}-1}\right)}}^{\infty} \bar{\mu}(\mathrm{d} x) \rho_{n_{l}, m_{l}}(x) \text { and } \\
& G\left(n_{l}, m_{l}\right)=\int_{r^{-m_{l} h\left(r^{m_{l}-1}\right)}}^{\infty} \mu(\mathrm{d} x) \rho_{n_{l}, m_{l}}(x) .
\end{aligned}
$$

The equality for $H\left(n_{l}, m_{l}\right)$ is evident since the random variable $\bar{I}_{m}^{(k)}$ is independent from $\left\{\Gamma_{m_{l}},\left(\bar{I}_{\left(j+1, m_{l}\right)}, R_{\left(j+1, m_{l}\right)} ; n_{l} \leq j \leq m_{l}-2\right)\right\}$. To show the second one, we use (2.13) in the following form

$$
I\left(\bar{\xi}^{(j+1)}\right)=\bar{I}_{\left(j+1, m_{l}\right)}+R_{\left(j+1, m_{l}\right)} I\left(\bar{\xi}^{\left(m_{l}\right)}\right)
$$

and the independence between $I\left(\bar{\xi}^{\left(m_{l}\right)}\right)$ and $\left\{\Gamma_{m_{l}},\left(\bar{I}_{\left(j+1, m_{l}\right)}, R_{\left(j+1, m_{l}\right)} ; n_{l} \leq j \leq m_{l}-2\right)\right\}$. In particular, it follows that for $l$ sufficiently large

$$
H\left(n_{l}, m_{l}\right) \geq \rho_{n_{l}, m_{l}}(N) \int_{N}^{\infty} \bar{\mu}(\mathrm{d} x) \quad \text { for } \quad N \geq r C
$$

where $C=\sup _{x \leq \beta} x^{-1} h(x)$.

Since $H\left(n_{l}, m_{l}\right)$ converges to 0 , as $l$ goes to $+\infty$ and $\bar{\mu}$ does not depend on $l$, then $\rho_{n_{l}, m_{l}}(N)$ also converges to 0 when $l$ goes to $+\infty$, for every $N \geq r C$.

On the other hand, we have

$$
G\left(n_{l}, m_{l}\right) \leq \rho_{n_{l}, m_{l}}(N) \int_{0}^{N} \mu(\mathrm{d} x)+\int_{N}^{\infty} \mu(\mathrm{d} x),
$$

then, letting $l$ and $N$ go to infinity, we get that $G\left(n_{l}, m_{l}\right)$ goes to 0 .

Note that the set $C_{n_{l}}$ satisfies

$$
\mathbb{P}\left(C_{n_{l}}\right) \geq 1-\mathbb{P}\left(r^{j+1} I\left(\bar{\xi}^{(j+1)}\right) \geq h\left(r^{j}\right), \text { for all } n_{l} \leq j \leq m_{l}-1\right)
$$

and it is not difficult to see that

$$
\mathbb{P}\left(r^{j+1} I\left(\bar{\xi}^{(j+1)}\right) \geq h\left(r^{j}\right), \text { for all } n_{l} \leq j \leq m_{l}-1\right) \leq \mathbb{P}\left(\Gamma_{m_{l}} \leq k r^{m_{l}}\right)+G\left(n_{l}, m_{l}\right)
$$

Then,

$$
\mathbb{P}\left(C_{n_{l}}\right) \geq \mathbb{P}\left(\Gamma_{m_{l}}>k r^{m_{l}}\right)-G\left(n_{l}, m_{l}\right),
$$

and since $\mathbb{P}\left(\Gamma_{m_{l}}>k r^{m_{l}}\right)=\mathbb{P}(\Gamma>k r)>0$ (see Lemma 1 and the properties of $\Gamma$ in Section 1), we conclude that $\lim \mathbb{P}\left(C_{n}\right)>0$ and with this we finish the proof.

For the integral test at $+\infty$, we define $\mathcal{H}_{\infty}^{-1}$ the totality of positive increasing functions $h(x)$ on $(0, \infty)$ that satisfy 
i) $\lim _{x \rightarrow+\infty} h(x)=+\infty$, and

ii) there exists $\beta>1$ such that $\sup _{x>\beta} x^{-1} h(x)<\infty$.

Theorem 4 Let $h \in \mathcal{H}_{\infty}^{-1}$.

i) If

$$
\int^{+\infty} \bar{F}_{\nu}\left(\frac{h(x)}{x}\right) \frac{\mathrm{d} x}{x}<\infty
$$

then for all $\epsilon>0$

$$
\mathbb{P}(U(x)<(1-\epsilon) h(x), \text { i.o., as } x \rightarrow+\infty)=0 .
$$

ii) If

$$
\int^{+\infty} \bar{F}\left(\frac{h(x)}{x}\right) \frac{\mathrm{d} x}{x}=\infty
$$

then for all $\epsilon>0$

$$
\mathbb{P}(U(x)<(1+\epsilon) h(x), \text { i.o., as } x \rightarrow+\infty)=1 .
$$

Proof: The proof is very similar to that in Theorem 3. First, note that we have the same results as Lemma 1 for $x$ large (see Corollary 3 of Chaumont and Pardo [9]), then we get the integral test following the same arguments for the proof of $(i)$ and $(i i)$ for the sequence $x_{n}=r^{n}$, for $r>1$, and noticing that if we define

$$
\begin{aligned}
C_{n} & =\left\{U(x)<h_{r}(x), \text { for some } x \in\left(r^{n},+\infty\right)\right\} \\
& =\left\{J_{t}^{(0)}>h_{r}^{-1}(t), \text { for some } t \in\left(U\left(r^{n}\right),+\infty\right)\right\},
\end{aligned}
$$

where $h_{r}(t)=r^{2} h(t)$, then the event $C=\cap_{n \geq 1} C_{n}$ is in the upper-tail sigma-field $\cap_{t} \sigma\left\{X_{s}^{(0)}: s \geq t\right\}$ which is trivial.

\section{Proof of Theorems 1 and 2}

Proof of Theorem 1: Let $\left(x_{n}\right)$ be a decreasing sequence which converges to 0 . We define the events $A_{n}=\left\{\right.$ There exists $t \in\left[U\left(x_{n+1}\right), U\left(x_{n}\right)\right]$ such that $\left.J_{t}^{(0)}>h(t)\right\}$. From the fact that $U\left(x_{n}\right)$ tends to 0 , a.s. when $n$ goes to $+\infty$, we see

$$
\left\{J_{t}^{(0)}>h(t) \text {, i.o., as } t \rightarrow 0\right\}=\limsup _{n \rightarrow+\infty} A_{n} .
$$

Since $h$ is an increasing function and $J_{U\left(x_{n}\right)}^{(0)} \geq x_{n}$ a.s., the following inclusions hold

$$
\left\{x_{n}>h\left(U\left(x_{n}\right)\right)\right\} \subset A_{n} \subset\left\{x_{n}>h\left(U\left(x_{n+1}\right)\right)\right\} .
$$


Now, we prove the convergent part. We choose $x_{n}=r^{n}$, for $r<1$ and $h_{r}(t)=r^{-2} h(t)$. Since $h$ is increasing, we deduce that

$$
\sum_{n} \mathbb{P}\left(r^{n}>h_{r}\left(U\left(r^{n+1}\right)\right)\right) \leq-\frac{1}{\log r} \int_{0}^{r} \mathbb{P}(t>h(U(t))) \frac{\mathrm{d} t}{t} .
$$

Replacing $h$ by $h_{r}$ in (3.14), we see that we can obtain our result if

$$
\int_{0}^{r} \mathbb{P}(t>h(U(t))) \frac{\mathrm{d} t}{t}<\infty .
$$

From elementary calculations, we deduce that

$$
\int_{0}^{r} \mathbb{P}(t>h(U(t))) \frac{\mathrm{d} t}{t}=\mathbb{E}\left(\int_{0}^{h^{-1}(r)} \mathbb{I}_{\{t / r<\nu I(\hat{\xi})<t / h(t)\}} \frac{\mathrm{d} t}{t}\right),
$$

where $h^{-1}(s)=\inf \{t>0, h(t)>s\}$, the right inverse function of $h$. Then, this integral converges if

$$
\int_{0}^{h^{-1}(r)} \mathbb{P}\left(\nu I(\hat{\xi})<\frac{t}{h(t)}\right) \frac{\mathrm{d} t}{t}<\infty
$$

This proves part $(i)$.

Next, we prove the divergent case. We suppose that $h$ satisfies

$$
\int_{0^{+}} \bar{F}\left(\frac{t}{h(t)}\right) \frac{\mathrm{d} t}{t}=\infty .
$$

Take, again, $x_{n}=r^{n}$, for $r<1$ and note that,

$$
\begin{aligned}
B_{n} & =\bigcup_{m=n}^{\infty} A_{m}=\left\{\text { There exist } t \in\left(0, U\left(r^{n}\right)\right] \text { such that } J_{t}^{(0)}>h_{r}(t)\right\} \\
& =\left\{\text { There exist } x \in\left(0, r^{n}\right] \text { such that } U(x)<h_{r}^{-1}(x)\right\}
\end{aligned}
$$

where $h_{r}(t)=r h(t)$ and $h_{r}^{-1}$ its right inverse function. Hence, by analogous arguments to the proof of Theorem 3 part $(i i)$ it is enough to prove that $\lim \mathbb{P}\left(B_{n}\right)>0$ to obtain our result. With this purpose, we will follow the proof of Theorem 3.

From inclusion (3.14) and the a.s. inequality in (1.10) we see

$$
\mathbb{P}\left(B_{n}\right) \geq 1-\mathbb{P}\left(r^{j} \leq r h\left(r^{j} I\left(\bar{\xi}^{(j)}\right)\right), \text { for all } n \leq j \leq m-1\right),
$$

where $m$ is chosen arbitrarily $m \geq n+1$.

Now, we define the events

$$
C_{n}=\left\{r^{n}>r h\left(r^{n} I\left(\bar{\xi}^{(n)}\right)\right)\right\}
$$

and we will prove that $\sum \mathbb{P}\left(C_{n}\right)=\infty$. Since the function $h$ is increasing, it is straightforward that

$$
\sum_{n \geq 1} \mathbb{P}\left(C_{n}\right) \geq \int_{0}^{+\infty} \mathbb{P}\left(r^{t}>h\left(r^{t} I(\hat{\xi})\right)\right) \mathrm{d} t=-\frac{1}{\log r} \int_{0}^{1} \mathbb{P}(t>h(t I(\hat{\xi}))) \frac{\mathrm{d} t}{t} .
$$


Hence, it is enough to prove that this last integral is infinite. In this direction, we have that

$$
\int_{0}^{r} \mathbb{P}(t>h(t I(\hat{\xi}))) \frac{\mathrm{d} t}{t}=\mathbb{E}\left(\int_{0}^{h^{-1}(r)} \mathbb{I}_{\{t / r<I(\hat{\xi})<t / h(t)\}} \frac{\mathrm{d} t}{t}\right) .
$$

On the other hand, we see that

$$
\begin{aligned}
\int_{0}^{h^{-1}(r)} \mathbb{P}\left(I(\hat{\xi})<\frac{t}{h(t)}\right) \frac{\mathrm{d} t}{t}= & \int_{0}^{h^{-1}(r)} \mathbb{P}\left(\frac{t}{r}<I(\hat{\xi})<\frac{t}{h(t)}\right) \frac{\mathrm{d} t}{t} \\
& +\int_{0}^{h^{-1}(r)} \mathbb{P}\left(I(\hat{\xi})<\frac{t}{r}\right) \frac{\mathrm{d} t}{t}
\end{aligned}
$$

and since $e^{-1} \hat{T}_{1} \leq I(\hat{\xi})$ a.s., then

$$
\begin{aligned}
\int_{0}^{h^{-1}(r)} \mathbb{P}\left(I(\hat{\xi})<\frac{t}{r}\right) \frac{\mathrm{d} t}{t} & \leq \mathbb{E}\left(\log ^{+} \frac{h^{-1}(r)}{r} I(\hat{\xi})^{-1}\right) \\
& \leq 1+\log ^{+} \frac{h^{-1}(r)}{r}+\mathbb{E}\left(\left|\log \hat{T}_{1}\right|\right)
\end{aligned}
$$

which is clearly finite from our assumptions. Then, we deduce that

$$
\mathbb{E}\left(\int_{0}^{h^{-1}(r)} \mathbb{I}_{\{t / r<I(\hat{\xi})<t / h(t)\}} \frac{\mathrm{d} t}{t}\right)=\infty,
$$

and hence $\sum \mathbb{P}\left(C_{n}\right)=\infty$.

Next following the same notation as in the proof of Theorem 3, we define the following events. For $n \leq m-1$

$$
D_{(n, m)}=\left\{r^{j} \leq r h\left(r^{j} \bar{I}_{(j, m)}\right), \text { for all } n \leq j \leq m-1\right\}
$$

and, for $r<k<1$ and $n \leq m-2$

$$
\begin{aligned}
& E_{n, m-1}=\left\{r^{j} \leq r h\left(r^{j} \bar{I}_{(j, m-1)}+r^{j} R_{(j, m-1)} \bar{I}_{(m-1, m)}\right), \text { for all } n \leq j \leq m-2\right\} \quad \text { and } \\
& E_{n, m-1}^{(k)}=\left\{r^{j} \leq r h\left(r^{j} \bar{I}_{(j, m-1)}+r^{j} R_{(j, m-1)} \bar{I}_{m-1}^{(k)}\right), \text { for all } n \leq j \leq m-2\right\} .
\end{aligned}
$$

Again, we have that

$$
D_{(n, m)}=E_{(n, m-1)} \bigcap\left\{r^{m-1} \leq r h\left(r^{m-1} \bar{I}_{(m-1, m)}\right)\right\} \bigcap\left\{\Gamma_{m-1}>r^{m} k\right\} .
$$

Now, for $n \leq m-1$, we define

$$
H(n, m)=\mathbb{P}\left(E_{(m, m-1)}^{(k)}, r^{m-1} \leq r h\left(r^{m-1} \bar{I}_{(m-1, m)}\right), \Gamma_{m-1}>r^{m} k\right) .
$$

Since $k>r$, we deduce that $\mathbb{P}\left(D_{(n, m)}\right) \geq H(n, m)$. Then similarly as in the proof of Theorem 3, we will prove that there exist $\left(n_{l}\right)$ and $\left(m_{l}\right)$, two increasing sequences such 
that $0 \leq n_{l} \leq m_{l}-1$, and $n_{l}, m_{l}$ go to $+\infty$ and $H\left(n_{l}, m_{l}\right)$ tends to 0 as $l$ goes to infinity.

We suppose the contrary, i.e., there exist $\delta>0$ such that $H(n, m) \geq \delta$ for all sufficiently large integers $m$ and $n$, hence

$$
\begin{aligned}
1 & \geq \mathbb{P}\left(\bigcup_{m=n+1}^{\infty} C_{m}\right) \geq \sum_{m=n+1}^{\infty} \mathbb{P}\left(C_{m} \bigcap\left(\bigcap_{j=n}^{m-1} C_{j}^{c}\right)\right) \\
& \geq \sum_{m=n+1}^{\infty} \mathbb{P}\left(r^{m}>\operatorname{rh}\left(r^{m} I\left(\bar{\xi}^{(m)}\right)\right)\right) \mathbb{P}\left(D_{(n, m)}\right) \\
& \geq \sum_{m=n+1}^{\infty} \mathbb{P}\left(r^{m}>r h\left(r^{m} I\left(\bar{\xi}^{(m)}\right)\right)\right) H(n, m) \geq \delta \sum_{m=n+1}^{\infty} \mathbb{P}\left(C_{m}\right),
\end{aligned}
$$

but since $\sum \mathbb{P}\left(C_{n}\right)$ diverges, we see that our assertion is true.

Next, we define

$$
\rho_{n_{l}, m_{l}}(x)=\mathbb{P}\left(\bigcap_{j=n_{l}}^{m_{l}-2}\left\{r^{j} \leq r h\left(r^{j} \bar{I}_{(j, m-1)}+r^{j} R_{(j, m-1)} x\right)\right\}, \Gamma_{m_{l}-1}>k r^{m_{l}-1}\right)
$$

and

$$
G\left(n_{l}, m_{l}\right)=\mathbb{P}\left(\bigcap_{j=n_{l}}^{m_{l}-1}\left\{r^{j} \leq r h\left(r^{j} I\left(\bar{\xi}^{(j)}\right)\right)\right\}, \Gamma_{m_{l}-1}>k r^{m_{l}-1}\right) .
$$

Since $h$ is increasing, we see that $\rho_{n_{l}, m_{l}}(x)$ is increasing in $x$.

Again, we express $H\left(n_{l}, m_{l}\right)$ and $G\left(n_{l}, m_{l}\right)$ as follows

$$
\begin{aligned}
& H\left(n_{l}, m_{l}\right)=\int_{0}^{+\infty} \bar{\mu}(\mathrm{d} x) \mathbb{I}_{\left\{h\left(r^{m_{l}-1} x\right) \geq r^{m_{l}}\right\}} \rho_{n_{l}, m_{l}}(x) \quad \text { and } \\
& G\left(n_{l}, m_{l}\right)=\int_{0}^{+\infty} \mu(\mathrm{d} x) \mathbb{I}_{\left\{h\left(r^{m_{l}-1} x\right) \geq r^{m_{l}}\right\}} \rho_{n_{l}, m_{l}}(x) .
\end{aligned}
$$

In particular, we get that for $l$ sufficiently large

$$
H\left(n_{l}, m_{l}\right) \geq \rho_{n_{l}, m_{l}}(N) \int_{N}^{+\infty} \bar{\mu}(\mathrm{d} x) \rho_{n_{l}, m_{l}}(x) \quad \text { for } \quad N \geq r C,
$$

where $C=\sup _{x \leq \beta} x / h(x)$. Hence following the same arguments of the proof of Theorem 3 , it is not difficult to see that $G\left(n_{l}, m_{l}\right)$ goes to 0 as $l$ goes to infinity and that

$$
\lim _{l \rightarrow+\infty} 1-\mathbb{P}\left(r^{j+1} \leq r h\left(r^{j+1} I\left(\bar{\xi}^{(j+1)}\right)\right), \text { for all } n_{l} \leq j \leq m_{l}-1\right)>0 .
$$

Then, we conclude that $\lim \mathbb{P}\left(B_{n}\right)>0$ and with this we finish the proof.

Proof of Theorem 2: We first consider the case where $x=0$. In this case the proof of the tests at $+\infty$ is almost the same as that of the tests at 0 . It is enough to apply the 
same arguments to the sequence $x_{n}=r^{n}$, for $r>1$.

Now, we prove $(i)$ for any $x>0$. Let $h \in \mathcal{H}_{\infty}$ such that $\int^{+\infty} \bar{F}_{\nu}\left(\frac{t}{h(t)}\right) \frac{\mathrm{d} t}{t}$ is finite. Let $x>0$ and $S_{x}=\inf \left\{t \geq 0: X_{t}^{(0)} \geq x\right\}$ and note by $\mu_{x}$ the law of $X_{S_{x}}^{(0)}$. Since clearly

$$
\int^{+\infty} \bar{F}_{\nu}\left(\frac{t}{h\left(t-S_{x}\right)}\right) \frac{\mathrm{d} t}{t}<\infty
$$

from the Markov property at time $S_{x}$, we have for all $\epsilon>0$

$$
\begin{aligned}
& \mathbb{P}_{0}\left(J_{t}>(1+\epsilon) h\left(t-S_{x}\right), \text { i. o., as } t \rightarrow \infty\right) \\
= & \int_{[x,+\infty)} \mathbb{P}_{y}\left(J_{t}>(1+\epsilon) h(t), \text { i. o., as } t \rightarrow \infty\right) \mu_{x}(\mathrm{~d} y)=0 .
\end{aligned}
$$

If $x$ is an atom of $\mu_{x}$, then equality (3.15) shows that

$$
\mathbb{P}\left(J_{t}^{(x)}>(1+\epsilon) h(t), \text { i. o., as } t \rightarrow \infty\right)=0
$$

and the result is proved. Suppose that $x$ is not an atom of $\mu_{x}$. Recall from Section 1, that $\log \left(x_{1}^{-1} \Gamma\right)$ is the limit in law of the overshoot process $\hat{\xi}_{\hat{T}_{x}}-x$, as $x \rightarrow+\infty$. So, it follows from [7], Theorem 1 that $X_{S_{x}}^{(0)} \stackrel{(d)}{=} \frac{x x_{1}}{\Gamma}$, and since $\mathbb{P}(\Gamma>z)$ for $z<x_{1}$, we have for any $\alpha>0, \mu_{x}(x, x+\alpha)>0$. Hence (3.15) shows that there exists $y>x$ such that

$$
\mathbb{P}\left(J_{t}^{(y)}>(1+\epsilon) h(t), \text { i. o., as } t \rightarrow \infty\right)=0,
$$

for all $\epsilon>0$. The previous allows us to conclude part $(i)$.

Part (ii) can be proved in the same way.

In some cases, it will prove complicated to find sharp estimations of the tail probability of $\nu I(\hat{\xi})$, given that we will not have enough information about the distribution of $\nu$. However, if we can determine the law of $I(\hat{\xi})$ then, by (1.2) we will also determine the law of $X_{1}^{(0)}$ and sometimes it will be possible to have sharp estimations of its tail probability. For this reason, we will give another integral test for the convergence cases in Theorems 1 and 2, in terms of the tail probability of $X_{1}^{(0)}$. Let us define

$$
G(t)=\mathbb{P}_{0}\left(X_{1}>t\right) .
$$

Corollary $1 \quad$ i) Let $h \in \mathcal{H}_{0}$. If

$$
\int_{0^{+}} G\left(\frac{h(t)}{t}\right) \frac{\mathrm{d} t}{t}<\infty,
$$

then for all $\epsilon>0$

$$
\mathbb{P}\left(J_{t}^{(0)}>(1+\epsilon) h(t), \text { i.o., as } t \rightarrow 0\right)=0 .
$$


ii) Let $h \in \mathcal{H}_{\infty}$. If

$$
\int^{+\infty} G\left(\frac{h(t)}{t}\right) \frac{\mathrm{d} t}{t}<\infty
$$

then and for all $\epsilon>0$

$$
\mathbb{P}\left(J_{t}^{(x)}>(1+\epsilon) h(t), \text { i.o., as } t \rightarrow \infty\right)=0 .
$$

Proof: The proof of this Corollary is consequence of the following inequality. By the scaling property,

$$
\bar{F}_{\nu}(t / h(t))=\mathbb{P}(U(1)<t / h(t))=\mathbb{P}\left(J_{1}^{(0)}>h(t) / t\right) \leq \mathbb{P}_{0}\left(X_{1}>h(t) / t\right),
$$

and then applying Theorem 1 part (i), we obtain the desired result.

In the same way, we can obtain another integral test for the convergence cases in Theorem 3 and 4, in terms of $G$.

Corollary 2 i) Let $h \in \mathcal{H}_{0}^{-1}$. If

$$
\int_{0^{+}} G\left(\frac{x}{h(x)}\right) \frac{\mathrm{d} x}{x}<\infty
$$

then for all $\epsilon>0$

$$
\mathbb{P}(U(x)<(1-\epsilon) h(x), \text { i.o., as } x \rightarrow 0)=0 .
$$

ii) Let $h \in \mathcal{H}_{\infty}^{-1}$. If

$$
\int^{+\infty} G\left(\frac{x}{h(x)}\right) \frac{\mathrm{d} x}{x}<\infty
$$

then for all $\epsilon>0$

$$
\mathbb{P}(U(x)<(1-\epsilon) h(x), \text { i.o., as } x \rightarrow \infty)=0 .
$$

\section{The regular case}

The first type of tail behaviour of $I(\hat{\xi})$ and $\nu I(\hat{\xi})$ that we consider is the case where $\bar{F}$ and $\bar{F}_{\nu}$ satisfy

$$
c t^{\alpha} L(t) \leq \bar{F}(t) \leq \bar{F}_{\nu}(t) \leq C t^{\alpha} L(t) \quad \text { as } \quad t \rightarrow 0,
$$

where $\alpha>0, c$ and $C$ are two positive constants such that $c \leq C$ and $L$ is a slowly varying function at 0 . An important example included in this case is when $\bar{F}$ and $\bar{F}_{\nu}$ are regularly varying functions at 0 .

The "regularity" of the behaviour of $\bar{F}$ and $\bar{F}_{\nu}$ gives us the following integral tests. 
Theorem 5 Under condition (4.16), the lower envelope of $U$ at 0 and at $+\infty$ is as follows:

i) Let $h \in \mathcal{H}_{0}^{-1}$, such that either $\lim _{x \rightarrow 0} h(x) / x=0$ or $\liminf _{x \rightarrow 0} h(x) / x>0$, then

$$
\mathbb{P}(U(x)<h(x), \text { i.o., as } x \rightarrow 0)=0 \text { or } 1
$$

according as

$$
\int_{0^{+}} \bar{F}\left(\frac{h(x)}{x}\right) \frac{\mathrm{d} x}{x} \quad \text { is finite or infinite. }
$$

ii) Let $h \in \mathcal{H}_{\infty}^{-1}$, such that either $\lim _{x \rightarrow+\infty} h(x) / x=0$ or $\liminf _{x \rightarrow+\infty} h(x) / x>0$, then

$$
\mathbb{P}(U(x)<h(x), \text { i.o., as } x \rightarrow \infty)=0 \text { or } 1,
$$

according as

$$
\int^{+\infty} \bar{F}\left(\frac{h(x)}{x}\right) \frac{\mathrm{d} x}{x} \quad \text { is finite or infinite. }
$$

Proof: First let us check that under condition (4.16) we have

$$
\int_{0}^{\lambda} \bar{F}_{\nu}\left(\frac{h(x)}{x}\right) \frac{\mathrm{d} x}{x}<\infty \quad \text { if and only if } \quad \int_{0}^{\lambda} \bar{F}\left(\frac{h(x)}{x}\right) \frac{\mathrm{d} x}{x}<\infty .
$$

Since $\nu I \leq I$ a.s., it is clear that we only need to prove that

$$
\int_{0}^{\lambda} \bar{F}\left(\frac{h(x)}{x}\right) \frac{\mathrm{d} x}{x}<\infty \quad \text { implies that } \int_{0}^{\lambda} \bar{F}_{\nu}\left(\frac{h(x)}{x}\right) \frac{\mathrm{d} x}{x}<\infty .
$$

From the hypothesis, either $\lim _{x \rightarrow 0} h(x) / x=0$ or $\liminf _{x \rightarrow 0} h(x) / x>0$. In the first case, from condition (4.16) there exists $\lambda>0$ such that, for every $x<\lambda$

$$
c\left(\frac{h(x)}{x}\right)^{\alpha} L\left(\frac{h(x)}{x}\right) \leq F\left(\frac{h(x)}{x}\right) \leq C\left(\frac{h(x)}{x}\right)^{\alpha} L\left(\frac{h(x)}{x}\right) .
$$

Since, we suppose that $\int_{0}^{\lambda} F\left(\frac{h(x)}{x}\right) \frac{\mathrm{d} x}{x}$ is finite, then

$$
\int_{0}^{\lambda}\left(\frac{h(x)}{x}\right)^{\alpha} L\left(\frac{h(x)}{x}\right) \frac{\mathrm{d} x}{x}<\infty
$$

and again from condition (4.16), we get that $\int_{0}^{\lambda} F_{\nu}\left(\frac{h(x)}{x}\right) \frac{\mathrm{d} x}{x}$ is also finite. In the second case, since for any $0<\delta<\infty, \mathbb{P}(I(\hat{\xi})<\delta)>0$, and $\liminf _{x \rightarrow 0} h(x) / x>0$, we have for any $y$

$$
0<\mathbb{P}\left(I(\hat{\xi})<\liminf _{x \rightarrow 0} \frac{h(x)}{x}\right)<\mathbb{P}\left(I(\hat{\xi})<\frac{h(y)}{y}\right)
$$


Hence, since for every $t \geq 0, F(t) \leq F_{\nu}(t)$, we deduce that

$$
\int_{0}^{\lambda} F\left(\frac{h(x)}{x}\right) \frac{\mathrm{d} x}{x}=\int_{0}^{\lambda} F_{\nu}\left(\frac{h(x)}{x}\right) \frac{\mathrm{d} x}{x}=\infty .
$$

Now, let us check that for any constant $\beta>0$,

$$
\int_{0}^{\lambda} \bar{F}\left(\frac{h(x)}{x}\right) \frac{\mathrm{d} x}{x}<\infty \quad \text { if and only if } \quad \int_{0}^{\lambda} \bar{F}\left(\frac{\beta h(x)}{x}\right) \frac{\mathrm{d} x}{x}<\infty
$$

Again, from the hypothesis either $\lim _{x \rightarrow 0} h(x) / x=0$ or $\liminf _{x \rightarrow 0} h(x) / x>0$. In the first case, we deduce (4.19) from (4.16). In the second case, from (4.18) both of the integrals in (4.19) are infinite.

Next, it follows from Theorem 3 part $(i)$ and (4.17) that if $\int_{0^{+}} \bar{F}\left(\frac{h(x)}{x}\right) \frac{\mathrm{d} x}{x}$ is finite, then for all $\epsilon>0, \mathbb{P}(U(x)<(1-\epsilon) h(t)$, i.o., as $t \rightarrow 0)=0$. If $\int_{0^{+}} \bar{F}\left(\frac{h(x)}{x}\right) \frac{\mathrm{d} x}{x}$ diverges, then from Theorem 3 part $(i i)$ that for all $\epsilon>0, \mathbb{P}(U(x)<(1+\epsilon) h(t)$, i.o., as $t \rightarrow 0)=1$. Then (4.19) allows us to drop $\epsilon$ in this implications.

The tests at $+\infty$ are proven through the same way.

Similarly, we get the following result for the upper envelope of the future infimum.

Theorem 6 Under condition (4.16), the upper envelope of the future infimum at 0 and at $+\infty$ is as follows:

i) Let $h \in \mathcal{H}_{0}$, such that either $\lim _{t \rightarrow 0} t / h(t)=0$ or $\liminf _{t \rightarrow 0} t / h(t)>0$, then

$$
\mathbb{P}\left(J_{t}^{(0)}>h(t), \text { i.o., as } t \rightarrow 0\right)=0 \text { or } 1,
$$

according as

$$
\int_{0^{+}} \bar{F}\left(\frac{t}{h(t)}\right) \frac{\mathrm{d} t}{t}<\infty \quad \text { is finite or infinite. }
$$

ii) Let $h \in \mathcal{H}_{\infty}$, such that either $\lim _{t \rightarrow+\infty} t / h(t)=0$ or $\liminf _{t \rightarrow+\infty} t / h(t)>0$, then for all $x \geq 0$

$$
\mathbb{P}\left(J_{t}^{(x)}>h(t), \text { i.o., as } t \rightarrow \infty\right)=0 \text { or } 1,
$$

according as

$$
\int^{+\infty} \bar{F}\left(\frac{t}{h(t)}\right) \frac{\mathrm{d} t}{t}<\infty \quad \text { is finite or infinite. }
$$

Proof: We prove this result by following the same arguments as the proof of the previous Theorem.

An example of such a behaviour will be given in section 7 (Example 3). 


\section{The log regular case}

The second type of behaviour that we shall consider is when $\log \bar{F}$ and $\log \bar{F}_{\nu}$ are regularly varying at 0 , i.e

$$
-\log \bar{F}_{\nu}(1 / t) \sim-\log \bar{F}(1 / t) \sim \lambda t^{\beta} L(t), \quad \text { as } t \rightarrow+\infty
$$

where $\lambda>0, \beta>0$ and $L$ is a slowly varying function at $+\infty$. Define the function

$$
\psi(t) \stackrel{(\text { def) }}{=} \frac{t}{\inf \{s: 1 / \bar{F}(1 / s)>|\log t|\}}, \quad t>0, \quad t \neq 1
$$

Then the lower envelope of $U$ may be described as follows

Theorem 7 Under condition (5.20), the process $U$ satisfies the following law of the iterated logarithm:

$$
\liminf _{x \rightarrow 0} \frac{U(x)}{\psi(x)}=1, \quad \text { and } \quad \liminf _{x \rightarrow+\infty} \frac{U(x)}{\psi(x)}=1 \quad \text { a.s. }
$$

Proof: This Theorem is a consequence of Theorems 3 and 4, and it is proven in the same way as Theorem 4 in 9 , we only need to emphasize that we can replace $\log \bar{F}_{\nu}$ by $\log \bar{F}$, since they are asymptotically equivalent.

Similarly the upper envelope of the future infimum may be described as follows. Define the function

$$
\phi(t) \stackrel{(\text { def })}{=} t \inf \{s: 1 / \bar{F}(1 / s)>|\log t|\}, \quad t>0, \quad t \neq 1 .
$$

Theorem 8 Under condition (5.20), the future infimum process satisfies the following law of the iterated logarithm:

i)

$$
\limsup _{t \rightarrow 0} \frac{J_{t}^{(0)}}{\phi(t)}=1, \quad \text { almost surely. }
$$

ii) For all $x \geq 0$,

$$
\limsup _{t \rightarrow+\infty} \frac{J_{t}^{(x)}}{\phi(t)}=1, \quad \text { almost surely. }
$$

Proof: As the previous Theorem, the proof of this result follows from Theorems 1 and 2 , and we use exactly the same arguments of the proof of Theorem 4 in [9].

In the following section, we obtain sharp estimates for $\log \bar{F}$ and $\log \bar{F}_{\nu}$ which will give us an important application of this case. 


\subsection{The case when $\xi$ has finite exponential moments}

Throughout this section we will suppose that the Lévy process $\xi$ associated to the PSSMP $X^{(x)}$ by its Lamperti representation, has finite exponential moments of arbitrary positive order. This condition is satisfied, for example, when the jumps of $\xi$ are bounded from above by some fixed number, in particular when $\xi$ is a Lévy process with no positive jumps. Then, we have

$$
\mathbb{E}\left(e^{\lambda \xi_{t}}\right)=\exp \{t \psi(\lambda)\}<+\infty \quad t, \lambda \geq 0 .
$$

From Theorem 25.3 in Sato [15], we know that this hypothesis is equivalent to assume that the Lévy measure $\Pi$ of $\xi$ satisfies

$$
\int_{[1, \infty)} e^{\lambda x} \Pi(\mathrm{d} x)<+\infty \quad \text { for every } \lambda>0 .
$$

Under this hypothesis, Bertoin and Yor [5] give a formula for the negative moments of the exponential functional $I(\hat{\xi})$

$$
\mathbb{E}\left(I(\hat{\xi})^{-k}\right)=m \frac{\psi(1) \cdots \psi(k-1)}{(k-1) !} \quad \text { for } \quad k \geq 1
$$

where $m=\mathbb{E}\left(\xi_{1}\right)$ and with the convention that the right-hand side equals $m$ for $k=$ 1. Moreover they proved that if $\xi$ has no positive jumps, then $1 / I(\hat{\xi})$ admits some exponential moments, this means that the distribution of $I(\hat{\xi})$ is determined by its negative entire moments.

From the entrance law of $X^{(x)}$ at 0 (see (1.2)), and the above equality (5.22), we get the following formula for the positive moments of $X_{1}^{(0)}$,

$$
\mathbb{E}_{0}\left(X_{1}^{k}\right)=\frac{\psi(1) \cdots \psi(k)}{k !} \quad \text { for } \quad k \geq 1
$$

Now, if we suppose that the Laplace exponent $\psi$ is regularly varying at $+\infty$ with index $\beta \in(1,2)$, i.e. $\psi(x)=x^{\beta} L(x)$, where $L$ is a slowly varying function at $+\infty$; then from equation (5.22), we see

$$
\mathbb{E}\left(I(\hat{\xi})^{-k}\right)=m((k-1) !)^{\beta-1} L(1) \cdots L(k-1),
$$

and from (5.23),

$$
\mathbb{E}_{0}\left(X_{1}^{k}\right)=(k !)^{\beta-1} L(1) \cdots L(k) .
$$

In consequence, we can easily deduce that

$$
\mathbb{E}\left(\exp \left\{\lambda I^{-1}(\hat{\xi})\right\}\right)<+\infty \quad \text { and } \quad \mathbb{E}_{0}\left(\exp \left\{\lambda X_{1}\right\}\right)<+\infty \quad \text { for all } \lambda>0
$$

This allows us to apply the Kasahara's Tauberian Theorem (see Theorem 4.12.7 in Bingham et al. 6] ) and get the following estimate. 
Proposition 1 Let $I(\hat{\xi})$ be the exponential functional associated to the Lévy process $\xi$. Suppose that $\psi$, the Laplace exponent of $\xi$, varies regularly at $+\infty$ with index $\beta \in(1,2)$. Then

$$
-\log \mathbb{P}_{0}\left(X_{1}>x\right) \sim-\log \mathbb{P}(I(\hat{\xi})<1 / x) \sim(\beta-1) \overleftarrow{H}(x) \quad \text { as } \quad x \rightarrow+\infty,
$$

where

$$
\overleftarrow{H}(x)=\inf \{s>0, \psi(s) / s>x\} .
$$

Recall that if the process $\xi$ has no positive jumps then the fact that the Laplace exponent $\psi$ is regularly varying at $\infty$ with index $\beta \in(1,2)$ is equivalent to that $\xi$ satisfies the Spitzer's condition (see Proposition VII.6 in Bertoin [1]), this is

$$
\lim _{t \rightarrow 0} \frac{1}{t} \int_{0}^{t} \mathbb{P}\left(\xi_{s} \geq 0\right) \mathrm{d} s=\frac{1}{\beta} .
$$

Proof: As we see above, the moment generating functions of $I(\hat{\xi})^{-1}$ and $X_{1}^{(0)}$ are well defined for all $\lambda>0$. We will only prove the case of $I(\hat{\xi})^{-1}$, the proof of the estimate of the tail probability of $X_{1}^{(0)}$ is similar.

From the main result of Geluk [10, we know that if $\phi$ is a regularly varying function at $+\infty$ with index $\sigma \in(0,1)$, then the following are equivalent

$$
\begin{aligned}
& \left(\mathbb{E}\left(I(\hat{\xi})^{-n}\right) / n !\right)^{1 / n} \sim e^{\sigma} / \phi(n) \quad \text { as } n \rightarrow+\infty, \\
& \log \mathbb{E}\left(\exp \left\{\lambda I(\hat{\xi})^{-1}\right\}\right) \sim \sigma \bar{\phi}(\lambda) \quad \text { as } \lambda \rightarrow+\infty,
\end{aligned}
$$

where $\bar{\phi}(\lambda)=\inf \{s>0, \phi(s)>\lambda\}$.

If we have (ii), then a straightforward application of Kasahara's Tauberian Theorem gives us

$$
-\log \mathbb{P}\left(I(\hat{\xi})^{-1}>x\right) \sim(1-\sigma) \bar{\varphi}(x) \quad \text { as } x \rightarrow+\infty,
$$

where $\bar{\varphi}$ is the asymptotic inverse of $s / \phi(s)$. Therefore, it is enough to show (i) with $\phi(s)=s^{2} / \psi(s)$ to obtain the desired result.

Let us recall that if $\psi$ is regularly varying at $\infty$ with index $\beta$, it can be expressed as $\psi(x)=x^{\beta} L(x)$, where $L(x)$ is a slowly varying function. By the formula (5.22) of negative moments of $I(\hat{\xi})$ and the fact that $\psi$ is regularly varying, we have

$$
\left(\mathbb{E}\left(I(\hat{\xi})^{-n}\right) / n !\right)^{1 / n}=m^{1 / n}(n !)^{\frac{\beta-2}{n}} n^{\frac{1-\beta}{n}}(L(1) \cdots L(n-1))^{\frac{1}{n}},
$$

due to the fact that $(n !)^{1 / n} \sim n e^{-1}$ for $n$ sufficiently large, then

$$
\left(\mathbb{E}\left(I(\hat{\xi})^{-n}\right) / n !\right)^{1 / n} \sim\left(n e^{-1}\right)^{\beta-2} \exp \left\{\frac{1}{n} \sum_{k=1}^{n} \log L(k)-\frac{1}{n} \log L(n)\right\} .
$$


On the other hand, from the proof of Proposition 2 of Rivero [14] we have that

$$
\frac{1}{n} \sum_{k=1}^{n} \log L(k) \sim \log L(n) \quad \text { as } \quad n \rightarrow+\infty .
$$

This implies that

$$
\left(\mathbb{E}\left(I(\hat{\xi})^{-n}\right) / n !\right)^{1 / n} \sim e^{2-\beta} \frac{\psi(n)}{n^{2}} .
$$

This last relation proves the Proposition.

Since the tail probability of $I(\hat{\xi})^{-1}$ and $X_{1}^{(0)}$ have the same asymptotic behaviour, it is logical to think that the tail probability of $(\nu I(\hat{\xi}))^{-1}$ could have the same behaviour. The next Corollary confirms this last argument.

Corollary 3 Let $I(\hat{\xi})$ be the exponential functional associated to the Lévy process $\xi$. Suppose that $\psi$, the Laplace exponent of $\xi$, varies regularly at $+\infty$ with index $\beta \in(1,2)$.

Then

$$
-\log \mathbb{P}(\nu I(\hat{\xi})<1 / x) \sim(\beta-1) \overleftarrow{H}(x) \quad \text { as } \quad x \rightarrow+\infty,
$$

where

$$
\overleftarrow{H}(x)=\inf \{s>0, \psi(s) / s>x\} .
$$

Proof: Since $\nu I(\hat{\xi}) \leq I(\hat{\xi})$ a.s., then

$$
-\log \mathbb{P}(\nu I(\hat{\xi})<1 / x) \leq-\log \mathbb{P}(I(\hat{\xi})<1 / x) .
$$

On the other hand, from the scaling property and since $X_{1}^{(0)} \geq J_{1}^{(0)}$ a.s., we see

$$
-\log \mathbb{P}(\nu I(\hat{\xi})<1 / x)=-\log \mathbb{P}(U(1)<1 / x) \geq-\log \mathbb{P}_{0}\left(X_{1}>x\right) .
$$

Hence, from the estimate (5.24) we have that

$$
-\log \mathbb{P}(\nu I(\hat{\xi})<1 / x) \sim(\beta-1) \overleftarrow{H}(x) \quad \text { as } \quad x \rightarrow+\infty,
$$

and this finishes the proof.

These estimates will allow us to obtain laws of iterated logarithm for the last passage time process and for the future infimum process in terms of the following function.

Let us define the function

$$
h(t)=\frac{\log |\log t|}{\psi(\log |\log t|)} \quad \text { for } \quad t>1, \quad t \neq e .
$$

By integration by parts, we can see that the function $\psi(\lambda) / \lambda$ is increasing, hence it is straightforward that the function $t h(t)$ is also increasing in a neighbourhood of $\infty$. 
Corollary 4 If $\psi$ is regularly varying at $+\infty$ with index $\beta \in(1,2)$, then

$$
\liminf _{x \rightarrow 0} \frac{U(x)}{x h(x)}=(\beta-1)^{\beta-1} \quad \text { almost surely }
$$

and,

$$
\liminf _{x \rightarrow+\infty} \frac{U(x)}{x h(x)}=(\beta-1)^{\beta-1} \quad \text { almost surely. }
$$

Proof: It is enough to see that for $t$ sufficiently small and $t$ sufficiently large the functions $t h(t)$ and $\psi(t)$ are asymptotically equivalent, but this is clear from (5.20). Now, applying Theorem 7 we obtain the desired law of the iterated logarithm.

Let us define

$$
f(t)=\frac{\psi(\log |\log t|)}{\log |\log t|} \quad \text { for } \quad t>1, \quad t \neq e .
$$

Corollary 5 If $\psi$ is regularly varying at $+\infty$ with index $\beta \in(1,2)$, then

$$
\limsup _{t \rightarrow 0} \frac{J_{t}}{t f(t)}=(\beta-1)^{-(\beta-1)} \quad \text { almost surely }
$$

and,

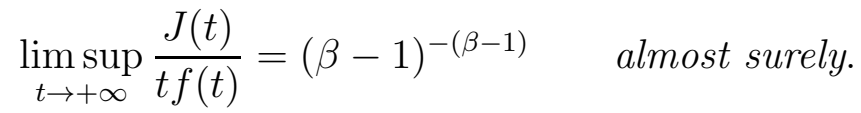

Proof: This proof follows from similar arguments of the last Corollary and using Theorem 8.

Example 1 Let $X_{t}^{(0)}$ be a stable Lévy process conditioned to stay positive with no positive jumps and with index $1<\alpha \leq 2$, (see Bertoin [1] for a proper definition). From Theorem VII.18 in [1], we know that the process time-reversed at its last passage time below $x,\left(x-X_{(U(x)-t)^{-}}^{(0)}, 0 \leq t \leq U(x)\right)$, has the same law as the killed process at its first passage time above $x,\left(\xi_{t}, 0 \leq t \leq T_{x}\right)$, where $\xi$ is a stable Lévy process with no positive jumps and with the same index as $X^{(0)}$.

From Theorem VII.1 in [1, we know that $\left(T_{x}, x \geq 0\right)$ is a subordinator with Laplace exponent $\Phi(\lambda)=\lambda^{1 / \alpha}$. Hence by the previous argument, we will have that $X^{(0)} \mathrm{drifts}$ towards $+\infty$ and that the process $(U(x), x \geq 0)$ is a stable subordinator with index $1 / \alpha$. Hence an application of the Tauberian theorem of de Brujin (see for instance Theorem 5.12.9 in Bingham et al. [6] ) gives us the following estimate

$$
-\log \bar{F}(x) \sim \frac{\alpha-1}{\alpha}\left(\frac{1}{\alpha}\right)^{1 /(\alpha-1)} x^{-1 /(\alpha-1)} \quad \text { as } \quad x \rightarrow 0 .
$$

Note that due to the absence of positive jumps $\nu=1$ a.s.

Then applying Theorems 7 and 8, we get the following law of the iterated logarithm. 
Corollary 6 Let $X^{(0)}$ be a stable Lévy process conditioned to stay positive with no positive jumps and $\alpha>1$. Then, its related last passage time process satisfies

$$
\liminf _{x \rightarrow 0} \frac{U(x)(\log |\log x|)^{\alpha-1}}{x^{\alpha}}=\frac{1}{\alpha}\left(1-\frac{1}{\alpha}\right)^{\alpha-1}, \quad \text { almost surely. }
$$

The same law of the iterated logarithm is satisfied for large times.

The future infimum process of $X^{(0)}$ also satisfies

$$
\limsup _{t \rightarrow 0} \frac{J_{t}^{(0)}}{t^{1 / \alpha}(\log |\log x|)^{1-1 / \alpha}}=\alpha(\alpha-1)^{-\frac{\alpha-1}{\alpha}}, \quad \text { almost surely, }
$$

and for all $x \geq 0$,

$$
\limsup _{t \rightarrow+\infty} \frac{J_{t}^{(x)}}{t^{1 / \alpha}(\log |\log x|)^{1-1 / \alpha}}=\alpha(\alpha-1)^{-\frac{\alpha-1}{\alpha}}, \quad \text { almost surely. }
$$

Example 2 Let us suppose that $\xi=\left(Y_{t}+c t, t \geq 0\right)$, where $Y$ is a stable Lévy process of index $\beta \in(1,2)$ with no positive jumps and $c$ a positive constant. Its Laplace exponent has the form

$$
\mathbb{E}\left(e^{\lambda \xi_{t}}\right)=\exp \left\{t\left(\lambda^{\beta}+c \lambda\right)\right\}, \quad \text { for } t \geq 0, \text { and } \lambda>0,
$$

where $c>0$. Note that under the hypothesis that $Y$ has no positive jumps, $\nu=1$ a.s. Let us define by $X^{(x)}$, the PSSMP associated to $\xi$ starting from $x$ and with scaling index $\alpha>0$, then when $x=0$ its last passage process $U$ satisfies

$$
\liminf _{x \rightarrow 0} \frac{U(x)}{x^{\alpha}(\log |\log x|)^{(1-\beta) \alpha}}=\alpha^{-\beta \alpha}(\beta-1)^{\alpha(\beta-1)}, \quad \text { almost surely. }
$$

We have the same law of the iterated logarithm at $+\infty$.

The future infimum process $J^{(x)}$ satisfies that

$$
\limsup _{t \rightarrow 0} \frac{J_{t}^{(0)}}{t^{\frac{1}{\alpha}}(\log |\log t|)^{\frac{(\beta-1)}{\alpha}}}=\alpha^{\frac{\beta}{\alpha}}(\beta-1)^{-\frac{\beta-1}{\alpha}}, \quad \text { almost surely, }
$$

and for all $x \geq 0$

$$
\limsup _{t \rightarrow+\infty} \frac{J_{t}^{(x)}}{t^{\frac{1}{\alpha}}(\log |\log t|)^{\frac{\beta-1}{\alpha}}}=\alpha^{\frac{\beta}{\alpha}}(\beta-1)^{-\frac{\beta-1}{\alpha}}, \quad \text { almost surely. }
$$

Note that when $\alpha=\beta$, the process $J$ has the same asymptotic behaviour as $\xi$, this is

$$
\limsup _{t \rightarrow 0(\text { or }+\infty)} \frac{\xi_{t}}{t^{\frac{1}{\beta}}(\log |\log t|)^{\frac{\beta-1}{\beta}}}=\beta(\beta-1)^{-\frac{\beta-1}{\beta}}, \quad \text { almost surely, }
$$

see Zolotarev [17] for details, and also the same asymptotic behaviour of $\bar{Y}$, the stable Lévy process conditioned to stay positive with no positive jumps (see Corollary 3). 


\section{Transient Bessel process.}

In this section we will suppose that $\xi=\left(2\left(B_{t}+a t\right), t \geq 0\right)$, where $B$ is a standard Brownian motion and $a>0$.

We define the process $Z=\left(Z_{t}, t \geq 0\right)$, the square of the $\delta$-dimensional Bessel processes starting at $x \geq 0$, as the unique strong solution of the stochastic differential equation

$$
Z_{t}=x+2 \int_{0}^{t} \sqrt{\left|Z_{s}\right|} \mathrm{d} \beta_{s}+\delta t, \quad \text { for } \quad \delta \geq 0
$$

where $\beta$ is a standard Brownian motion.

By the Lamperti representation, we know that we can define $X^{(x)}$ a PSSMP starting at $x>0$, such that

$$
X_{x I_{t}(\xi)}^{(x)}=x \exp \left\{\xi_{t}\right\} \quad \text { for } \quad t \geq 0 .
$$

Then, applying the Itô's formula and Dubins-Schwartz's Theorem (see for instance, Revuz and Yor [13]), we get

$$
X_{x I_{t}(\xi)}^{(x)}=x+2 \int_{0}^{x I_{t}(\xi)} \sqrt{X_{s}^{(x)}} \mathrm{d} B_{s}+2(a+1) x I_{t}(\xi) .
$$

Hence it follows that $X^{(x)}$ satisfies (6.29) with $\delta=2(a+1)$ and therefore $X^{(x)}$ is the square of the $\delta$-dimensional Bessel processes starting at $x>0$. From the main result of Bertoin and Yor 4, we may define $X^{(x)}$ at $x=0$, and we can computed its entrance law by (1.2). Since, we suppose that $a>0$, we deduce that $X^{(x)}$ is a transient process and that $\delta>2$.

From the formula of negative moments (5.22) of the exponential functional $I(\hat{\xi})$, we can deduce (see Example 3 in Bertoin and Yor [5]) the following identity in distribution

$$
\int_{0}^{\infty} \exp \left\{-2\left(B_{s}+a s\right)\right\} \mathrm{d} s \stackrel{(d)}{=} \frac{1}{2 \gamma_{a}}
$$

where $\gamma_{a}$ is a gamma random variable with index $a>0$. In fact, we can also deduce that $X_{1}^{(0)}$ is distributed as $2 \gamma_{a+1}$.

We recall that the distribution of $\gamma_{a}$ for $a>0$, is given by

$$
\mathbb{P}\left(\gamma_{a} \leq x\right)=\frac{1}{\Gamma(a)} \int_{0}^{x} e^{-y} y^{a-1} \mathrm{~d} y, \quad \text { where } \quad \Gamma(a)=\int_{0}^{\infty} e^{-y} y^{a-1} \mathrm{~d} y
$$

It is important to note that due the continuity of the paths of $X^{(0)}$, we have that $\nu=1$ almost surely.

The following Lemma will be helpful for the application of our general results to the case of transient Bessel processes.

Lemma 2 Let $a>0$, then there exist $c$ and $C$, two positive constants such that

$$
c e^{-x} x^{a-1} \leq \int_{x}^{\infty} e^{-y} y^{a-1} \mathrm{~d} y \leq C e^{-x} x^{a-1}, \quad \text { for } \quad x \geq \frac{C(a-1)}{C-1}
$$


From this Lemma, we deduce the following integral tests.

Theorem 9 Let $h \in \mathcal{H}_{0}^{-1}$, then:

i) If

$$
\int_{0^{+}}(x / 2 h(x))^{\frac{\delta-4}{2}} \exp \{-x / 2 h(x)\} \frac{\mathrm{d} x}{x}<\infty,
$$

then for all $\epsilon>0$

$$
\mathbb{P}(U(x)<(1-\epsilon) h(x), \text { i.o., as } x \rightarrow 0)=0 .
$$

ii) If

$$
\int_{0^{+}}(x / 2 h(x))^{\frac{\delta-4}{2}} \exp \{-x / 2 h(x)\} \frac{\mathrm{d} x}{x}=\infty,
$$

then for all $\epsilon>0$

$$
\mathbb{P}(U(x)<(1+\epsilon) h(x), \text { i.o., as } x \rightarrow 0)=1 .
$$

Proof: The proof of this Theorem follows from the fact that

$$
\mathbb{P}(I<x)=\mathbb{P}\left(\gamma_{(\delta-2) / 2}>1 / 2 x\right) \quad \text { for } \quad x>0,
$$

and an application of Theorem 3 and Lemma 2.

Theorem 10 Let $h \in \mathcal{H}_{\infty}^{-1}$, then:

i) If

$$
\int^{+\infty}(x / 2 h(x))^{\frac{\delta-4}{2}} \exp \{-x / 2 h(x)\} \frac{\mathrm{d} x}{x}<\infty,
$$

then for all $\epsilon>0$

$$
\mathbb{P}(U(x)<(1-\epsilon) h(x), \text { i.o., as } x \rightarrow+\infty)=0 .
$$

ii) If

$$
\int^{+\infty}(x / 2 h(x))^{\frac{\delta-4}{2}} \exp \{-x / 2 h(x)\} \frac{\mathrm{d} x}{x}=\infty,
$$

then for all $\epsilon>0$

$$
\mathbb{P}(U(x)<(1+\epsilon) h(x), \text { i.o., as } x \rightarrow+\infty)=1 .
$$


Proof: The proof of these integral tests is very similar to the proof of the previous result, it is enough to apply Lemma 2 and Theorem 4 to equality (6.32).

From these integral tests, we get the following law of iterated logarithm.

$$
\liminf _{x \rightarrow 0} U(x) \frac{2 \log |\log x|}{x}=1 \quad \text { and } \quad \liminf _{x \rightarrow+\infty} U(x) \frac{2 \log \log x}{x}=1 \quad \text { almost surely. }
$$

Note that we are also in the "logregular" case and we can apply Theorem 7 to get the same law of the iterated logarithm.

For the upper envelope of the future infimum process, we have the following integral tests.

Theorem 11 Let $h \in \mathcal{H}_{0}$, then:

i) If

$$
\int_{0^{+}}(h(t) / 2 t)^{\frac{\delta-4}{2}} \exp \{-h(t) / 2 t\} \frac{\mathrm{d} t}{t}<\infty,
$$

then for all $\epsilon>0$

$$
\mathbb{P}\left(J_{t}^{(0)}>(1+\epsilon) h(t), \text { i.o., as } t \rightarrow 0\right)=0 .
$$

ii) If

$$
\int_{0^{+}}(h(t) / 2 t)^{\frac{\delta-4}{2}} \exp \{-h(t) / 2 t\} \frac{\mathrm{d} t}{t}=\infty,
$$

then for all $\epsilon>0$

$$
\mathbb{P}\left(J_{t}^{(0)}>(1-\epsilon) h(t), \text { i.o., as } t \rightarrow 0\right)=1 .
$$

Proof: We get this result applying Theorem 1 and Lemma 2 to the equality (6.32).

Theorem 12 Let $h \in \mathcal{H}_{\infty}$, then for all $x \geq 0$ :

i) If

$$
\int^{+\infty}(h(t) / 2 t)^{\frac{\delta-4}{2}} \exp \{-h(t) / 2 t\} \frac{\mathrm{d} t}{t}<\infty,
$$

then for all $\epsilon>0$

$$
\mathbb{P}\left(J_{t}^{(x)}>(1+\epsilon) h(t), \text { i.o., as } t \rightarrow+\infty\right)=0 .
$$

ii) If

$$
\int^{+\infty}(h(t) / 2 t)^{\frac{\delta-4}{2}} \exp \{-h(t) / 2 t\} \frac{\mathrm{d} t}{t}=\infty,
$$

then for all $\epsilon>0$

$$
\mathbb{P}\left(J_{t}^{(x)}>(1-\epsilon) h(t), \text { i.o., as } t \rightarrow+\infty\right)=1 .
$$


Proof: The proof of these integral test is similar to the previous Theorem. We only replace Theorem 1 by Theorem 2 .

From these integral tests, we get the following law of iterated logarithm,

$$
\limsup _{t \rightarrow 0} \frac{J_{t}^{(0)}}{2 t \log |\log t|}=1 \quad \text { and } \quad \limsup _{t \rightarrow+\infty} \frac{J_{t}^{(x)}}{2 t \log \log t}=1 \quad \text { almost surely, }
$$

for $x \geq 0$. Here we can also obtain the same law of the iterated logarithm applying Theorem 8 .

\section{The upper envelope of increasing self-similar Markov processes.}

From the Lamperti representation (1.1), we know that increasing PSSMP are related to subordinators and by the main result of Bertoin and Caballero we can define this processes at 0 . In this section we suppose that $\xi$ is a subordinator.

Since the PSSMP $X^{(x)}$ is increasing, we know that its supremum, its past infimum and its future infimum are the same. Moreover, its first passage time over the level $y>0$ is the same as the last passage time below $y$. Therefore, with our previous main results we can describe the upper envelope of $X^{(x)}$ at 0 (when $x=0$ ) and at $+\infty$ (for all $x \geq 0$ ) and also the lower envelope of its first passage time defined by,

$$
S_{y}=\inf \left\{t: X_{t}^{(0)} \geq y\right\}, \quad \text { for } y>0 .
$$

From Proposition 2.1 in Carmona, Petit and Yor 8 provided that $m<\infty$, we know that the law of $I(\hat{\xi})$ admits a density $\rho$ which is infinitely differentiable on $(0,+\infty)$. Moreover, from (1.2) the entrance law has also a density and is described as follows

$$
\rho_{1}(x)=\frac{1}{m x} \rho\left(\frac{1}{x}\right) \quad \text { for } x \in(0, \infty) .
$$

Generally speaking, we cannot estimate the tail probability $\mathbb{P}(I(\hat{\xi})<t)$ for $t$ sufficiently small, so we will now give some examples in which we can obtain an estimation.

\subsection{Examples}

Example 1 Let $\xi=N$ be a standard Poisson process. From Proposition 3 in Bertoin and Yor [5], we know that

$$
-\log \mathbb{P}(I(\hat{\xi})<t) \sim \frac{1}{2}(\log 1 / t)^{2}, \quad \text { as } t \rightarrow 0,
$$

and also that

$$
-\log \rho(x) \sim \frac{1}{2}(\log 1 / x)^{2}, \quad \text { as } x \rightarrow 0 .
$$


From (7.33) we get that

$$
-\log \rho_{1}(x) \sim \frac{1}{2}(\log x)^{2}, \quad \text { as } x \rightarrow+\infty .
$$

Now, applying Theorem 4.12.10 in Bingham et al. [6] and doing some elementary calculations, we obtain that

$$
-\log \int_{x}^{+\infty} \rho_{1}(y) \mathrm{d} y \sim \frac{1}{2}(\log x)^{2} \quad \text { as } x \rightarrow+\infty .
$$

These estimations allow us the following laws of the iterated logarithm. Let us define

$$
f(t)=t \exp \{-\sqrt{2 \log |\log t|}\}
$$

Corollary 7 Let $N$ be a Poisson process, then the PSSMP $X^{(x)}$ associated to $N$ by the Lamperti representation satisfies the following law of the iterated logarithm,

$$
\limsup _{t \rightarrow 0} \frac{X_{t}^{(0)} f(t)}{t^{2}}=1, \quad \text { almost surely. }
$$

For all $x \geq 0$

$$
\limsup _{t \rightarrow+\infty} \frac{X_{t}^{(x)} f(t)}{t^{2}}=1, \quad \text { almost surely. }
$$

The first passage time process $S$ associated to $X^{(0)}$ satisfies the following law of the iterated logarithm,

$$
\liminf _{x \rightarrow 0} \frac{S_{x}}{f(x)}=1, \quad \text { and } \quad \liminf _{x \rightarrow+\infty} \frac{S_{x}}{f(x)}=1 \quad \text { a.s. }
$$

Proof: The proof of this Corollary is consequence of the previous estimations and application of the divergent parts of Theorems 1, 2, 3 and 4, and Corollaries 1 and 2.

Example 2 Let $\xi$ be a subordinator with zero drift and Lévy measure $\Pi(\mathrm{d} x)=$ $a b e^{-b x} \mathrm{~d} x$, with $a, b>0$, i.e. a compound Poisson process with jumps having an exponential distribution. Carmona, Petit and Yor showed that the density $\rho$ of $I(\hat{\xi})$ is given by

$$
\rho(x)=\frac{a^{1+b}}{\Gamma(1+b)} x^{b} e^{-a x}, \quad \text { for } x>0 .
$$

The PSSMP associated to $\xi$ by the Lamperti representation is the well-know generalized Watanabe process. From (17.33), we get that

$$
\mathbb{P}_{0}\left(X_{1}>y\right)=\frac{b a^{1+b}}{\Gamma(1+b)} \int_{0}^{1 / y} z^{b-1} e^{-a z} \mathrm{~d} z
$$


On the other hand, It is clear that

$$
\mathbb{P}(I(\xi)<y)=\frac{a^{1+b}}{\Gamma(1+b)} \int_{0}^{y} x^{b} e^{-a x} \mathrm{~d} x
$$

Elementary calculations give us the following inequality,

$$
e^{-a x} \frac{x^{b+1}}{b+1} \leq \int_{0}^{x} z^{b} e^{-a z} \mathrm{~d} z \leq x^{b} \frac{\left(1-e^{-a x}\right)}{a} .
$$

Hence for $x$ sufficiently small, there exists $c_{b}$ a positive constant such that

$$
\mathbb{P}(I(\xi)<x) \sim c_{b} \frac{a^{1+b}}{\Gamma(1+b)} x^{b+1} e^{-a x},
$$

and for $y$ sufficiently large there exist $C_{b}$ such that

$$
\mathbb{P}_{0}\left(X_{1}>y\right) \sim C_{b} \frac{a^{1+b}}{\Gamma(1+b)}(1 / y)^{b} e^{-a / y} .
$$

Then applying the divergent part of Theorems 1,2,3 and 4 and Corollaries 1 and 2, we get the following laws of the iterated logarithm for the generalized Watanabe process and its first passage time. Let us define

$$
g(t)=a^{-1} t \log |\log t| .
$$

Corollary 8 Let $\xi$ be a compound Poisson process with jumps having and exponential distribution as above, then the PSSMP $X^{(x)}$ associated to $\xi$ by the Lamperti representation satisfies the following law of the iterated logarithm,

$$
\limsup _{t \rightarrow 0} \frac{X_{t}^{(0)} g(t)}{t^{2}}=1, \quad \text { almost surely. }
$$

For all $x \geq 0$,

$$
\limsup _{t \rightarrow+\infty} \frac{X_{t}^{(x)} g(t)}{t^{2}}=1, \quad \text { almost surely. }
$$

The first passage time process $S$ associated to $X^{(0)}$ satisfies the following law of the iterated logarithm,

$$
\liminf _{x \rightarrow 0} \frac{S_{x}}{g(x)}=1, \quad \text { and } \quad \liminf _{x \rightarrow 0} \frac{S_{x}}{g(x)}=1 \quad \text { a.s. }
$$

Example 3 Let $\xi$ a subordinator with zero drift and Lévy measure

$$
\Pi(\mathrm{d} x)=\frac{\beta e^{x}}{\Gamma(1-\beta)\left(e^{x}-1\right)^{1+\beta}} \mathrm{d} x,
$$


with $\beta \in(0,1)$. The PSSMP $X^{(x)}$ associated to $\xi$ is the stable subordinator of index $\beta$ (see for instance Rivero [14]).

From Zolotarev [18, we know that there exists $k$ a positive constant such that

$$
\mathbb{P}_{0}\left(X_{1}>x\right) \sim k x^{-\beta} \quad x \rightarrow+\infty
$$

It is well-known that the law of $X_{1}^{(0)}$ has a density $\rho_{1}$ with respect to the Lebesgue measure and that this density is unimodal, i.e., there exist $b>0$ such that $\rho_{1}(x)$ is increasing in $(0, b)$ and decreasing in $(b,+\infty)$ (see for instance Sato [15]). Hence $\rho_{1}$ is monotone in a neighborhood of $+\infty$, then by the monotone density Theorem (see Theorem 1.7.2 in Bingham et al. [6] page 38) we get

$$
\rho_{1}(x) \sim k \beta x^{-\beta-1} \quad x \rightarrow+\infty .
$$

From (7.33), we can easily deduce that

$$
\rho(x) \sim m k \beta x^{\beta} \quad x \rightarrow 0
$$

and it is also easy to see that

$$
\mathbb{P}(I(\hat{\xi})<x) \sim m k \beta x^{\beta+1} \quad x \rightarrow 0 .
$$

Hence, we can apply Theorems 5 and 6 , and deduce the following results.

Corollary 9 Let $\xi$ be a subordinator without drift and such that its Lévy mesure $\Pi$ satisfies

$$
\Pi(\mathrm{d} x)=\frac{\beta e^{x}}{\Gamma(1-\beta)\left(e^{x}-1\right)^{1+\beta}} \mathrm{d} x .
$$

The lower envelope of $S$, the first passage time of the PSSMP $X^{(0)}$, at 0 and at $+\infty$ is as follows:

i) Let $h \in \mathcal{H}_{0}^{-1}$, such that either $\lim _{x \rightarrow 0} h(x) / x=0$ or $\liminf _{x \rightarrow 0} h(x) / x>0$, then

$$
\mathbb{P}\left(S_{x}<h(x), \text { i.o., as } x \rightarrow 0\right)=0 \text { or } 1,
$$

according as

$$
\int_{0^{+}}\left(\frac{h(x)}{x}\right)^{\beta+1} \frac{\mathrm{d} x}{x} \quad \text { is finite or infinite. }
$$

ii) Let $h \in \mathcal{H}_{\infty}^{-1}$, such that either $\lim _{x \rightarrow+\infty} h(x) / x=0$ or $\liminf _{x \rightarrow+\infty} h(x) / x>0$, then

$$
\mathbb{P}\left(S_{x}<h(x), \text { i.o., as } x \rightarrow \infty\right)=0 \text { or } 1,
$$

according as

$$
\int^{+\infty}\left(\frac{h(x)}{x}\right)^{\beta+1} \frac{\mathrm{d} x}{x} \quad \text { is finite or infinite. }
$$


Corollary 10 Let $\xi$ be a subordinator as in Corollary 8, the upper envelope of $X^{(x)}$ at $0(x=0)$ and at $+\infty(x \geq 0)$ is as follows:

i) Let $h \in \mathcal{H}_{0}$, such that either $\lim _{t \rightarrow 0} t / h(t)=0$ or $\liminf _{t \rightarrow 0} t / h(t)>0$, then

$$
\mathbb{P}\left(X_{t}^{(0)}>h(t), \text { i.o., as } t \rightarrow 0\right)=0 \text { or } 1,
$$

according as

$$
\int_{0^{+}}\left(\frac{x}{h(x)}\right)^{\beta+1} \frac{\mathrm{d} x}{x} \quad \text { is finite or infinite. }
$$

ii) Let $h \in \mathcal{H}_{\infty}$, such that either $\lim _{t \rightarrow+\infty} t / h(t)=0$ or $\liminf _{t \rightarrow+\infty} t / h(t)>0$, then for all $x \geq 0$

$$
\mathbb{P}\left(X_{t}^{(x)}>h(t), \text { i.o., as } t \rightarrow \infty\right)=0 \text { or } 1
$$

according as

$$
\int^{+\infty}\left(\frac{x}{h(x)}\right)^{\beta+1} \frac{\mathrm{d} x}{x} \quad \text { is finite or infinite. }
$$

Acknowledgements. I would like to thank Loïc Chaumont for guiding me through the development of this work, and for all his helpful advice. I would also like to thank Victor Rivero for all his useful suggestions. 


\section{References}

[1] J. Bertoin: Lévy processes. Cambridge University Press, Cambridge, (1996).

[2] J. Bertoin and M.E. Caballero: Entrance from 0+ for increasing semi-stable Markov processes. Bernoulli. 8, no. 2, 195-205, (2002).

[3] J. Bertoin And M. Yor: On subordinators, self-similar Markov processes and some factorizations of the exponential functional. Electron. Comm. Probab. 6, 95-106, (2001).

[4] J. Bertoin And M. Yor: The entrance laws of self-similar Markov processes and exponential functionals of Lévy processes. Potenctial Anal. 17, no. 4, 389-400, (2002).

[5] J. Bertoin And M. Yor: On the entire moments of self-similar Markov processes and exponential funtionals of Lévy processes. Ann. Fac. Sci. Toulouse VI Ser. Math. 11, no. 1, 33-45, (2002).

[6] N.H. Bingham, C.M. Goldie and J.L. Teugels: Regular Variation. Cambridge University Press, Cambridge, (1989)

[7] M.E. Caballero and L. Chaumont: Weak convergence of positive self-similar Markov processes and overshoots of Lévy processes. To appear in Annals of Probab. (2006).

[8] P. Carmona, F. Petit and M; Yor: On the distribution and asymptotic results for exponential funtionals of Lévy processes. In: M. Yor (editor) Exponential functionals and principal values related to Brownian motion. Biblioteca de la Revista Matemática Iberoamericana. 71-121, (1997).

[9] L. Chaumont and J.C. Pardo: The lower envelope of positive self-similar Markov processes. Prépublication No. 994 du Laboratoire de Probabilités et Modèles Aléatoires de l'université de Paris 6, (2005).

[10] J.L. GeLuK: On the relation between the tail probability and the moments of a random variable. Nederl. Aked. Wetensch. Indag. Math. 46, 401-405, (1984).

[11] D. Khoshnevisan, T.M. Lewis and W.V. Li: On the future infima of some transient processes. Probab. Theory Relat. Fields 99 337-360, (1994).

[12] J. Lamperti: Semi-stable Markov processes. I. Z. Wahrscheinlichkeitstheorie und Verw. Gebiete, 22, 205-225, (1972).

[13] D. Revuz And M. Yor: Continuous martingale and Brownian motion. Third edition. Springer-Verlag, Berlin, (1999)

[14] V. Rivero: A law of iterated logarithm for increasing self-similar Markov processes. Stoch. Stoch. Rep. 75, no. 6, 443-472, (2003). 
[15] K. Sato: Lévy processes and infinitely divisible distributions. Cambridge University Press, Cambridge, (1999).

[16] T. Watanabe: Sample function behavior of increasing processes of class L. Probab. Theory Relat. Fields 104, 349-374, (1996).

[17] V.M. Zolotarev: Analog of the iterated logarithm law for semi-continuous stable processes. Theory Probab. Appl. 9, 512-513, (1964).

[18] V.M. Zolotarev: One-dimensional stable distributions. American Mathematical Society, Providence. (1986) 